\title{
Scanning SQUID microscope system for geological samples: system integration and initial evaluation
}

\author{
Hirokuni Oda ${ }^{1 *} \mathbb{D}$, Jun Kawai ${ }^{2}$, Masakazu Miyamoto², Isoji Miyagi ${ }^{3}$, Masahiko Sato ${ }^{1}$, Atsushi Noguchi ", \\ Yuhji Yamamoto ${ }^{4}$, Jun-ichi Fujihira ${ }^{5}$, Nobuyoshi Natsuhara ${ }^{6}$, Yoshiyasu Aramaki ${ }^{7}$, Takashige Masuda ${ }^{8}$ \\ and Chuang Xuan ${ }^{9}$
}

\begin{abstract}
We have developed a high-resolution scanning superconducting quantum interference device (SQUID) microscope for imaging the magnetic field of geological samples at room temperature. In this paper, we provide details about the scanning SQUID microscope system, including the magnetically shielded box (MSB), the XYZ stage, data acquisition by the system, and initial evaluation of the system. The background noise in a two-layered PC permalloy MSB is approximately 40-50 pT. The long-term drift of the system is approximately $\geq 1 \mathrm{nT}$, which can be reduced by drift correction for each measurement line. The stroke of the XYZ stage is $100 \mathrm{~mm} \times 100 \mathrm{~mm}$ with an accuracy of $\sim 10 \mu \mathrm{m}$, which was confirmed by laser interferometry. A SQUID chip has a pick-up area of $200 \mu \mathrm{m} \times 200 \mu \mathrm{m}$ with an inner hole of $30 \mu \mathrm{m} \times 30 \mu \mathrm{m}$. The sensitivity is $722.6 \mathrm{nT}$. The flux-locked loop has four gains, i.e., $\times 1, \times 10, \times 100$, and $\times 500$. An analog-to-digital converter allows analog voltage input in the range of about $\pm 7.5 \mathrm{~V}$ in $0.6-\mathrm{mV}$ steps. The maximum dynamic range is approximately $\pm 5400 \mathrm{nT}$, and the minimum digitizable magnetic field is $\sim 0.9 \mathrm{pT}$. The sensor-to-sample distance is measured with a precision line current, which gives the minimum of $\sim 200 \mu \mathrm{m}$. Considering the size of pick-up coil, sensor-to-sample distance, and the accuracy of XYZ stage, spacial resolution of the system is $\sim 200 \mu \mathrm{m}$. We developed the software used to measure the sensor-to-sample distance with line scan data, and the software to acquire data and control the $X Y Z$ stage for scanning. We also demonstrate the registration of the magnetic image relative to the optical image by using a pair of point sources placed on the corners of a sample holder outside of a thin section placed in the middle of the sample holder. Considering the minimum noise estimate of the current system, the theoretical detection limit of a single magnetic dipole is $\sim 1 \times 10^{-14} \mathrm{Am}^{2}$. The new instrument is a powerful tool that could be used in various applications in paleomagnetism such as ultrafine-scale magnetostratigraphy and single-crystal paleomagnetism.
\end{abstract}

Keywords: SQUID sensor, Magnetic microscopy, Magnetic shield, Point source, XYZ stage, Sensitivity, Noise, Detection limit, Drift, Paleomagnetism, Magnetostratigraphy

\section{Introduction}

Magnetometers with a superconducting quantum interference device (SQUID) have been used for highsensitivity measurements in paleomagnetism. Superconducting rock magnetometers (SRMs) (manufactured by

\footnotetext{
*Correspondence: hirokuni-oda@aist.go.jp

1 Research Institute of Geology and Geoinformation, Geological Survey of Japan, AIST, Central 7, 1-1-1 Higashi, Tsukuba 305-8567, Japan Full list of author information is available at the end of the article
}

$2 G$ Enterprises) contributed significantly to the development of paleomagnetism and are the most well-known magnetometers. In addition to measurements of discrete paleomagnetic specimens, pass-through-type SRMs, which can resolve magnetization with a resolution of $\sim 2 \mathrm{~cm}$ after deconvolution, have been used extensively for continuous measurements of sediment long-cores and u-channels (e.g., Constable and Parker 1991; Jackson et al. 2010; Oda and Xuan 2014; Xuan and Oda 2015). A gradiometer-type SQUID sensor has been used to detect 
magnetic inclusions in volcanic ash particles contained in natural ice to increase the spacial resolution of continuous measurements (Oda et al. 2016a).

On the other hand, scanning magnetic microscopy allows the mapping of magnetic fields with high spatial resolution and sensitivity. It has been used increasingly in the study of meteorites (Weiss et al. 2000; Fu et al. 2014), volcanic rocks (Weiss et al. 2007b), impacted rocks using laboratory laser experiments (Gattacceca et al. 2006), and sulfur-bearing metamorphic rocks (Fischer et al. 2014). Different techniques use different magnetic sensors, including the SQUID microscope (e.g., Fong et al. 2005; Weiss et al. 2007a, b), the magnetic tunnel junction microscope (e.g., Lima et al. 2014), and the magnetic microscope with nitrogen vacancy (NV) quantum diamond (Fu et al. 2014). SQUID microscopy is a powerful technique for imaging weak magnetic field distributions with the highest field sensitivity. A SQUID microscope allows samples of about $100 \mu \mathrm{m}$ to be scanned at room temperature (Kirtley and Wikswo 1999; Chatraphorn et al. 2000; Ono and Ishiyama 2004; Fong et al. 2005). An important use of this technique is the study of geological samples (Fong et al. 2005; Baudenbacher et al. 2002, 2003; Wang et al. 2014; Weiss et al. 2000, 2007a, b; Oda et al. 2011). In a recent work, SQUID microscope made it possible to use magnetostratigraphic dating as a way to image ultrafine-scale magnetic stripes related to geomagnetic reversals preserved in ferromanganese crust samples (Oda et al. 2011).

A single mineral crystal such as zircon with magnetic inclusions has been used to measure natural remanent magnetization (e.g., Sato et al. 2015; Tarduno et al. 2015), which could be used to determine the magnetic field of the Earth in the distant past (Tarduno et al. 2015). The practical limit of a normal-type DC SQUID rock magnetometer is $4 \times 10^{-12} \mathrm{Am}^{2}$ (Sato et al. 2015), whereas that of an improved magnetometer with a smaller bore of $6.2 \mathrm{~mm}$ is an order of magnitude smaller (Tarduno et al. 2015). Recently, Fu et al. (2016) used a scanning SQUID microscope to measure the magnetic moment of a set of single zircon crystals to estimate paleointensity.

Basically, current collection of scanning SQUID microscopes used for paleomagnetic studies on geological samples were developed based on the model described in Fong et al. (2005). In order to realize extensive application of ultrafine-scale magnetostratigraphy of geological samples such as ferromanganese crusts for dating with a scanning SQUID microscope and pursue the possibility to improve for better resolution, sensitivity, convenience, and maintainability, we have decided to construct a scanning SQUID microscope with some new ideas. This article presents the details of a recently developed SQUID microscope system for imaging geological samples and our evaluation of the SQUID microscope system. We also demonstrate a new way to register a magnetic image relative to the optical image using a pair of point sources.

\section{Scanning SQUID microscope system}

The scanning SQUID microscope system at the Geological Survey of Japan (GSJ), AIST, is shown in Fig. 1. It includes the SQUID microscope (Fig. 1a-d, D), the $X Y Z$ stage (Fig. 1a, b, C), the magnetically shielded box (MSB; Fig. 1b-f, E), and other system components. The SQUID microscope fits in the upper part of the MSB, which is supported by an aluminum frame (Fig. 1a-d, B). Figure 1a is a front view of the system, with the large front access door (Fig. 1a-c, A) closed, whereas Fig. 1d is a photograph taken from the front when the front access door is removed for maintenance. Figure $1 b, c$ shows the side view and the top view of the system, respectively. Figure 1e shows the top door, which is open for the transfer of liquid He into the system, and Fig. If indicates the main door, which is open to set a sample for measurements. Figure 2 shows all of the remaining components of the SQUID microscope system. The flux-locked loop (FLL) linearizes and amplifies the SQUID signal, which is digitized by an analog-to-digital converter (ADC) in the $X Y Z$ stage controller. The $X Y Z$ stage controller, which is operated by a PC, also controls the $X Y Z$ stage.

\section{SQUID microscope}

The cryostat and the SQUID microscope were described in detail by Kawai et al. (2016). In order to simplify the structure and reduce the work for operations, we designed the cryostat without liquid $\mathrm{N}_{2}$ reservoir. Alternatively, we designed the cryostat with low boil-off rate of liquid helium without liquid nitrogen, optimizing the thermal shield configuration including superinsulations. The exterior of the cryostat is aluminum, except for the bottom flange, which is glass fiber-reinforced plastic (GFRP). The cylindrical 10-L liquid He reservoir is made of GFRP and has a hollow center. A rigid GFRP shaft that passes through the hollow center directly connects a micrometer spindle attached to the top flange of the cryostat to a copper rod placed beneath the He reservoir via thermal anchors. Instead, Fong et al. (2005) used a lever mechanism for sensor-to-sample distance adjustment, which is manipulated from outside of the cryostat. A SQUID chip is mounted on the conical top of a sapphire rod, which is tightly connected to the copper rod. By rotating the micrometer spindle, the copper rod and the SQUID chip move up and down in the range of $\sim 1 \mathrm{~mm}$ with an accuracy of $\sim 5 \mu \mathrm{m}$. The SQUID chip was electrically connected to silver-based thin-film electrodes using silver paste. In order to achieve the minimum sensor-to-sample distance, it is important to minimize the 


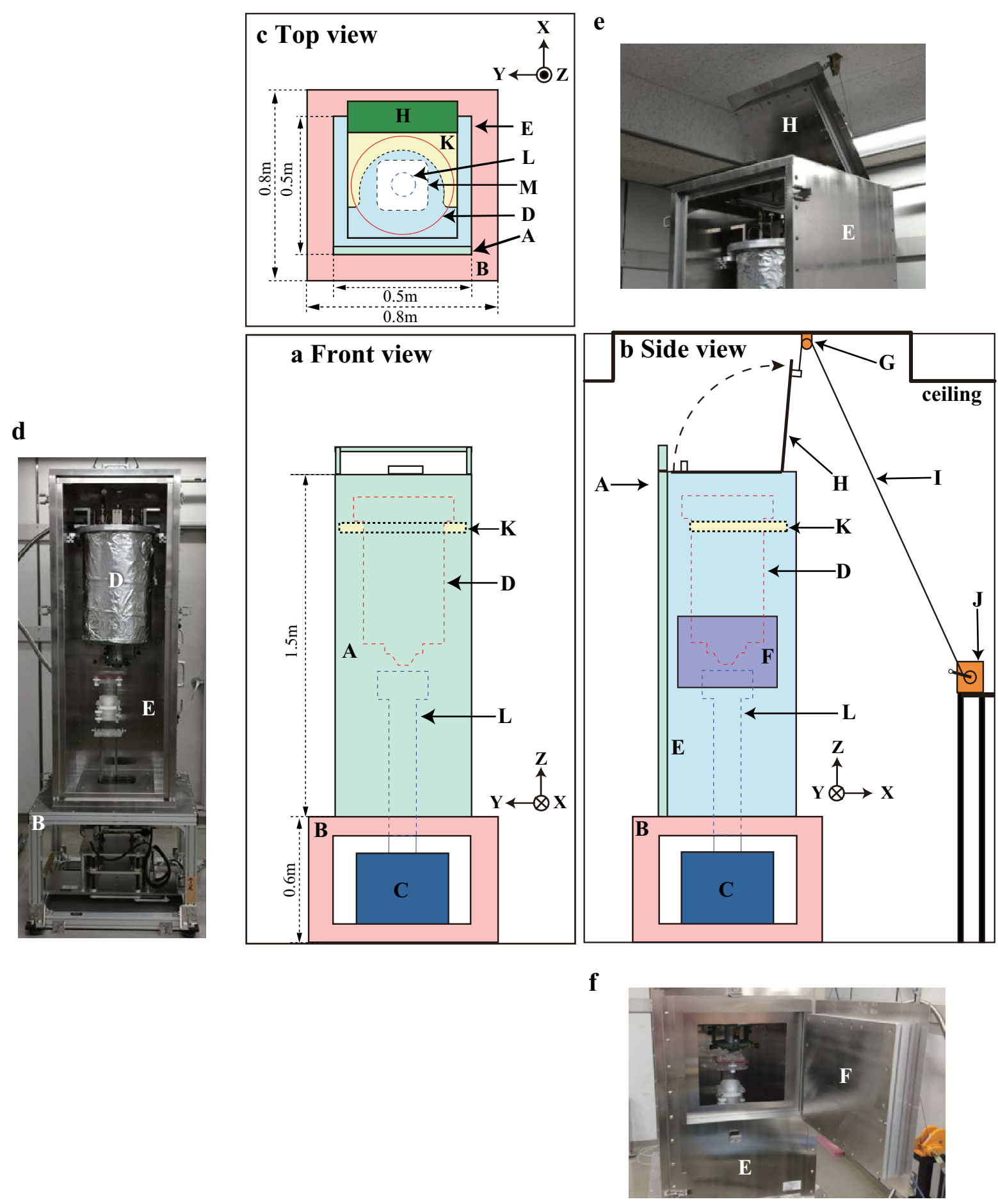

Fig. 1 Schematic diagram of the magnetically shielded box (MSB) and main system components. The MSB has three access doors. a Front view; A (light green): large front access, which is used for maintenance of the SQUID microscope, $B$ (pink): aluminum frame that supports the MSB, C (blue): stepping motor components for XYZ stage, D (red lines): SQUID microscope. b Side view; $E$ (light blue): main body of the MSB, $F$ (purple): main door for sample access, $G$ (orange): pulley attached to the ceiling, $H$ (dark green): top door for maintenance and liquid He transfer, l: wire, J (orange): manual winch fixed to the floor and the wall. c Top view; K (yellow): U-shaped support for the SQUID microscope, L (blue lines): non-magnetic long acrylic pipe to support sample holder, M: opening to allow the pipe to move. $\mathbf{d}$ Front view photograph of the MSB $(E)$ on the aluminum frame (B) without a large front access together with the SQUID microscope $(D)$ and the XYZ stage. e Photograph of the top door $(H)$ for the MSB. $\mathbf{f}$ Photograph of the main door $(F)$ for the MSB $(E)$ 


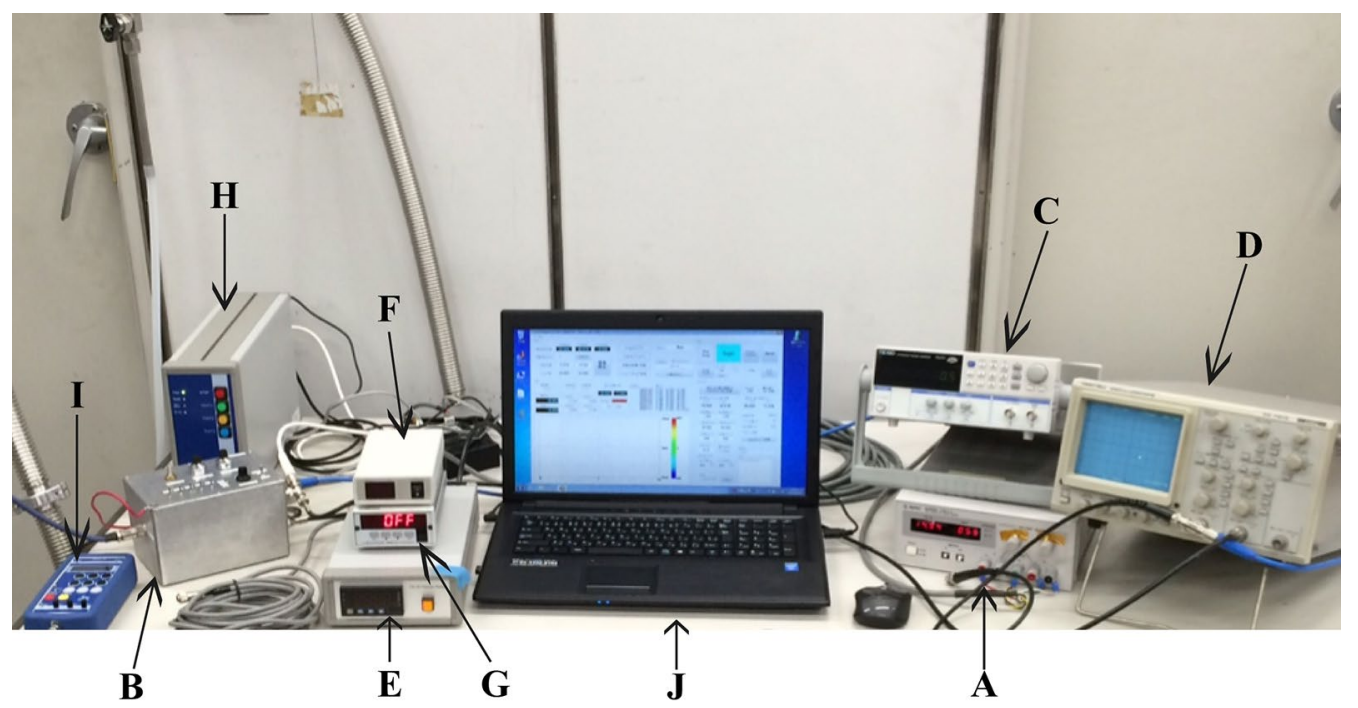

Fig. 2 Auxiliary system components: A: power supply, B: flux-locked loop (FLL), C: function generator, D: oscilloscope, E: level meter, F: flow meter, G: precision current supply (Model 121 Programmable DC Current source, LakeShore Cryotronics, Inc.), H: XYZ stage controller, I: remote controller, J: control PC

height of silver paste mound. On the other hand, Fong et al. (2005) glued SQUID chip on the sapphire rod, grinded and polished the edges, deposited silver pads to the sides, then gold wires were attached to the silver pads on the sides. Our method allows to change SQUID chip for the maintenance easily in case of SQUID malfunction, etc.

The inner vacuum space is separated from the outside by a thin sapphire window. The original $40-\mu \mathrm{m}$-thick, 3-mm-diameter sapphire window (Kawai et al. 2016) was replaced with a $50-\mu \mathrm{m}$-thick, 4 -mm-diameter sapphire window for better visibility. The sapphire window is attached to a GFRP cone, which is fixed to the bottom flange through an aluminum bellows. By moving the bellows using vertical and horizontal screws, the SQUID chip is positioned roughly with respect to the sapphire window before precise adjustments are made using the micrometer spindle. The SQUID is a simple washer-type magnetometer fabricated on a $1 \mathrm{~mm} \times 1 \mathrm{~mm}$ silicon substrate. The washer is $200 \mu \mathrm{m} \times 200 \mu \mathrm{m}$ with an inner hole of $30 \mu \mathrm{m} \times 30 \mu \mathrm{m}$. The SQUID chip is glued onto the tip of the sapphire rod and electrically connected to the electrodes patterned on the rod. We used a low-drift FLL to allow measurements with low-frequency drift noise up to several tens of hours. The field noise was $1.1 \mathrm{pT} / \mathrm{Hz}^{1 / 2}$ at $1 \mathrm{~Hz}$, and the low-frequency temperature drift was $\sim 10 \mathrm{pT} /{ }^{\circ} \mathrm{C}$. The liquid He boil-off rate was reduced significantly from the initial value of $3.1 \mathrm{~L} /$ day (Kawai et al. 2016) to $2.5 \mathrm{~L} /$ day after improving the thermal insulation. The SQUID microscope can operate stably for about
4 days on $10 \mathrm{~L}$ of liquid He before the reservoir becomes empty.

\section{Magnetically shielded box}

The scanning SQUID microscope is located in a MSB made of two-layered PC permalloy (Fig. 1b-f, E). There is a large door on the front of the MSB (Fig. 1a-c, A) through which the SQUID microscope can be removed for maintenance if necessary. The MSB is supported by an aluminum frame underneath it (Fig. 1a-d, B), which holds the driving components of the $X Y Z$ stage. An aluminum support holds the SQUID microscope in place in the MSB (Fig. 1a-c, K). Figure $1 \mathrm{~b}$ is a side view of the MSB with a sample access door (F), with its hinge on the right-hand side (see Fig. 1f). The sample access door is tightly locked via the handles at the top and bottom of the door to prevent leakage of the magnetic field lines. The door on the top of the MSB (Fig. 1b-d, H) can be opened to transfer liquid $\mathrm{He}$, for maintenance, and for fine-tuning of the sensor-to-sample distance with a micrometer spindle. The top door is hinged to the top of the MSB (see Fig. 1b) and can be opened and closed by using a winch (Fig. 1b, J) with stainless-steel wire (Fig. 1b, I), which is going through a pulley attached to the laboratory ceiling (Fig. 1b, G). The top door can also be tightly locked via the handles on its two unhinged sides. There is a rounded square-shaped hole at the bottom of the MSB (Fig. 1c, M), within which a cylinder (Fig. 1c, L) supporting the sample holder moves while scanning with the $X Y Z$ stage (Fig. 1a, $b, C)$. Right before installation, the magnetic field in the 
center of the MSB was measured using a Helmholtz coil (an approximately 2-m $\times 2$-m rectangle). With the Helmholtz coil, a magnetic field of $0.1-\mathrm{Hz}$ sine wave was generated at amplitudes of $500 \mathrm{nTpp}$ and the magnetic field was measured at the center of the MSB with a three-axis fluxgate magnetometer (Model FM-3500, MIT Co. Ltd.) capable of measuring AC magnetic field up to $1 \mathrm{kHz}$. The results were analyzed, and the shielding factors in the $X$, $Y$, and $Z$ directions were calculated as $\sim 1 / 257, \sim 1 / 288$, and $\sim 1 / 91$, respectively. After installation of the MSB, the inner wall was demagnetized with a demagnetizing coil. With a three-axis fluxgate sensor (Model 520A, Applied Physics Systems, Inc.), the residual magnetic field at the measurement position was measured as $2.3,-3.0,3.7 \mathrm{nT}$ in $X$-, $Y$ - and $Z$ - axis, respectively .

\section{$X Y Z$ stage and controller}

The $X Y Z$ stage, which is located under the SQUID microscope in the center of the MSB, is shown in Figs. 1c and 3 (schematic diagram). The main function of the $X Y Z$ stage is to move a thin section sample smoothly and precisely in the $X$ and $Y$ directions. Because it is important to reduce the $\mathrm{DC}$ and $\mathrm{AC}$ magnetic fields produced by the $X Y Z$ stage as much as possible, we used three stepping motors covered by magnetic shield enclosures made of $\mathrm{PC}$ permalloy for $\mathrm{X}$-, Y-, and Z- axes movement. Once the $\mathrm{Z}$-axis motor is set up and fixed, the position of the $\mathrm{X}$ - and Y- axes motors do not move relative to the SQUID microscope during measurements. Unlike the motors and the magnetic shields, most of the components of the support and the $X Y Z$ stage were made of non-magnetic material such as aluminum. The distance between the sensor and the base of the non-magnetic cylinder (Fig. 3, G), where the stepping motors are located, is $\sim 80 \mathrm{~cm}$. All the motors are on the other side of the opening at the bottom of the MSB (Fig. 2c, K) to reduce magnetic noise. The $Z$-axis motor (Fig. 3, A) drives four shafts (C) connected by a timing belt (B). The rotation of the shafts in two directions moves the main support (D) up and down. The $X Y$-moving table (G) is above the support and is driven by the $X$-axis motor (E) and the $Y$-axis motor (F). The non-magnetic acrylic acid resin cylinder $(\mathrm{H})$ is in between the $X Y$-moving table $(\mathrm{G})$ and the $X Y Z$ stage $(\mathrm{K})$, to which it is connected by a manual height adjuster (I) and three tilt (and fine height) adjusters (J). A holding block (L) is attached to the $X Y Z$ stage $(\mathrm{K})$ and supports the sample holder $(\mathrm{M})$ with two rubber bands. A thin section $(\mathrm{N})$ is held in a rectangular shallow pit of the sample holder which is pressed to one of the corners by a rubber cylinder pushing the diagonal corner and is covered with a thin protective film (O). The sapphire window (P) of the SQUID microscope then presses down on the sample. The $X Y Z$ stage can be controlled either by a remote controller or by software on

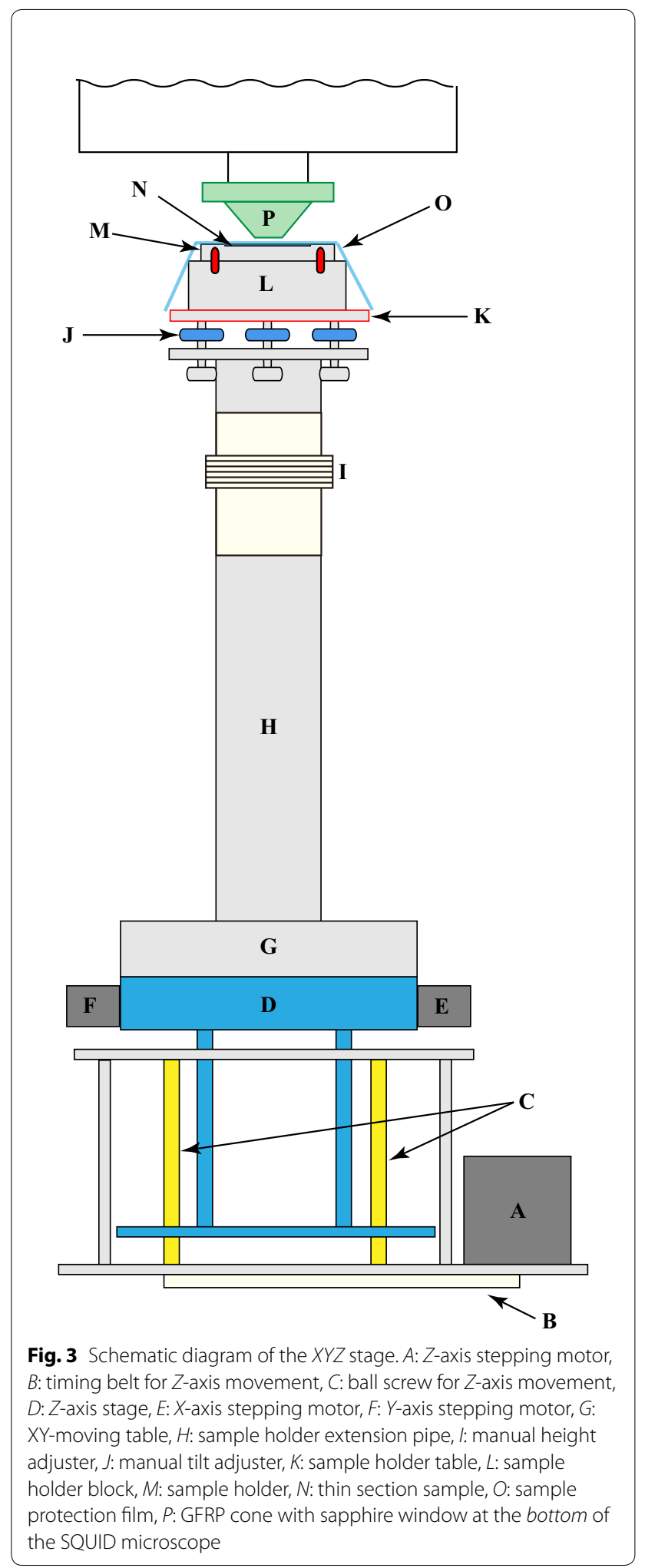

a PC through serial interface. The strokes in the $X$ and $Y$ directions are $100 \mathrm{~mm}$, each with an approximate accuracy of $10 \mu \mathrm{m}$. This is discussed in the following section. 


\section{Electrical system components and interfaces}

Figure 4 shows a schematic block diagram of the electrical components of the SQUID system. The SQUID sensor of the SQUID microscope (a) is connected to an FLL (b), which has its own power supply (c). Before any measurements are made, the "Tune" switch is turned on and the function generator (d) is used to tune the SQUID sensor while an oscilloscope (e) monitors the waveform. The $X Y Z$ stage controller contains an ADC (f) for digitizing the voltage output from the FLL and a motor controller $(\mathrm{g})$ that drives the stepping motors (i). The $X Y Z$ stage controller is connected to a PC (j) via a USB cable through which the command sequence is given to conduct the measurements and retrieve the data. The stepping motors can also be controlled with a remote controller (h). Measurement data are acquired using a 16-bit ADC chip, where $\pm 5 \mathrm{~V}$ input range corresponds to 14 bits with 1 LSB (least significant bit) of $0.61 \mathrm{mV}$. In reality, the ADC can properly convert voltage between -7.593 and $+8.633 \mathrm{~V}$, which corresponds approximately to -5.8 and $+6.2 \mu \mathrm{T}$ with the postamplifier gain of 1 .

A flow sensor ( $k$ ) between the SQUID microscope and the He gas outlet detects the flow of He gas, which is monitored by a flow meter (l). In addition, a He level meter $(\mathrm{m})$ monitors the level of liquid $\mathrm{He}$. The inset in the upper-right corner of Fig. 4 shows the line current calibration system. The line current (n) is produced by the precision current supply (o), which is further explained in the following section.

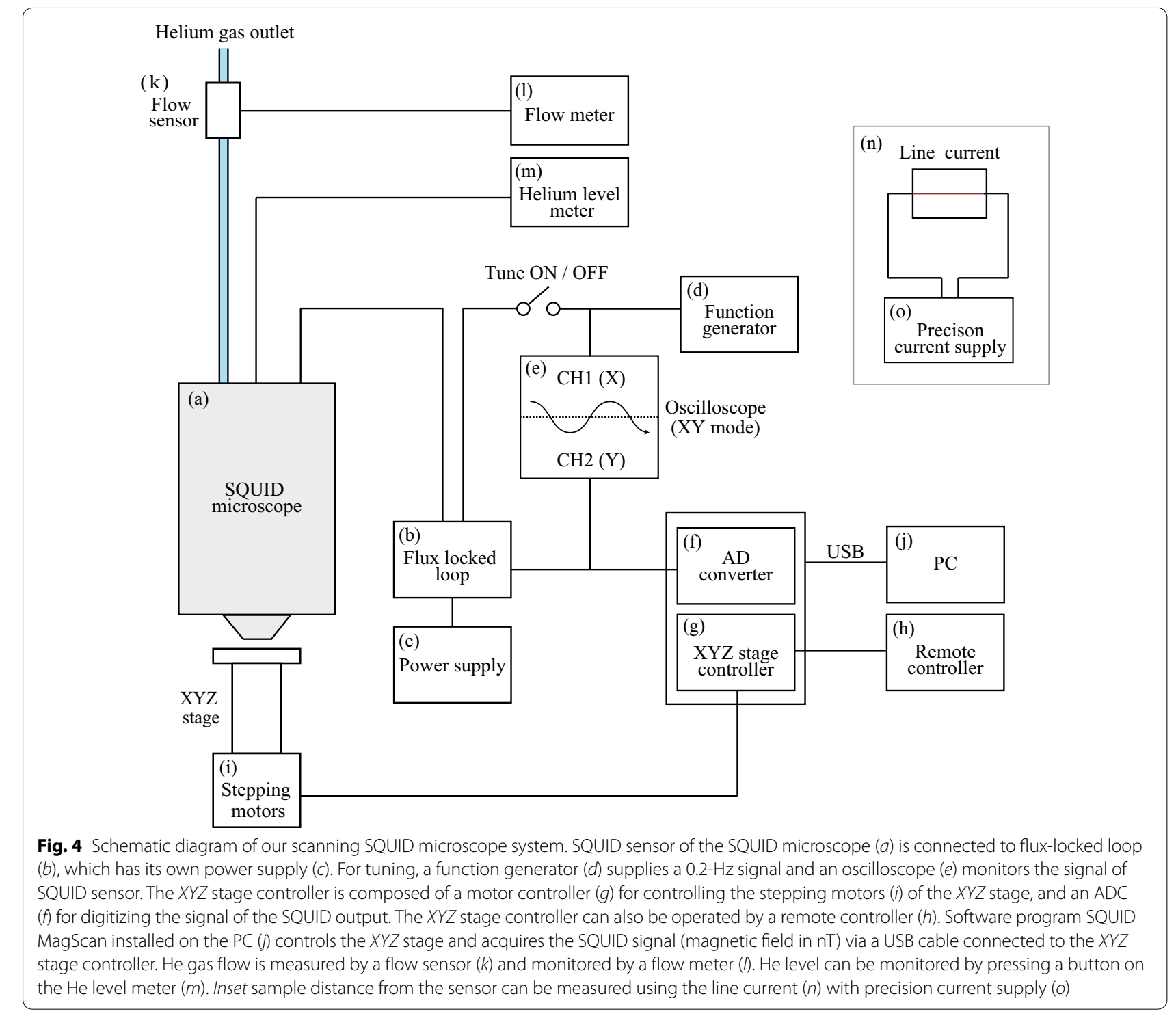




\section{SQUID MagScan software}

Figure 5 shows a screen image from the SQUID microscope measurement and control software SQUID MagScan. The software allows the operator to move the $X$ - and $Y$-axes stepping motors of the $X Y Z$ stage. However, the $Z$-axis stepping motor is controlled only by the remote controller to avoid the possibility of breaking the sapphire window. The operator must register the home position of the SQUID system before starting a scan of a thin section sample. In addition, parameters such as step $(\mathrm{mm})$, length $(\mathrm{mm})$, and speed $(\mathrm{mm} / \mathrm{min})$ for the $X$ - and $Y$-axes must be entered along with the delay [seconds (s)] between the time that the stepping motors stop and the measurement begins and the number of repeat measurements. A scan consists of sequential stepwise movement in the $+Y$ direction and data acquisition followed by similar movement in $+X$ direction. The operator can choose one of two scanning modes, i.e., meander (alternate between $+Y$ and $-Y$ directions) and one way (always in $+Y$ direction). The analog output voltage of the FLL is digitized by the ADC of the $X Y Z$ stage controller. The gain of the measurement $(\times 1, \times 10, \times 100$, or $\times 500)$ is also input to the controller. The data from each scan are stored in a data file that contains the serial number, positions in $X$ and $Y$ directions, voltage, converted magnetic field values (calculated using the conversion factor and the gain), and the time stamp. During a scan, the magnetic field values are color-coded and displayed in the panel in the lowerleft corner of the MagScan user interface. Minimum and maximum values of the voltage and the magnetic field are displayed above the panel. If the analog voltage from the FLL exceeds the limit allowed by the ADC, the Message box displays "overscaled" in red and the message remains until the end of the scan.

\section{Calibration and evaluation Calibration of a SQUID chip}

Figure 6a shows the experimental setup, including a Circular Current Array System (Higuchi et al. 1989; Yoshida et al. 1994; Adachi et al. 2014), for the calibration of a SQUID sensor. The measurements were made in a magnetically shielded room of two-layered PC permalloy with a shielding factor of $1 / 100$ at $1 \mathrm{~Hz}$. The Circular Current Array System is also used to determine the sensitivity and positioning of the SQUID sensor array used for magnetoencephalography or magnetospinography (Adachi et al. 2014). The Circular Current Array System used in this study has the same setup as that shown in Fig. 1 of

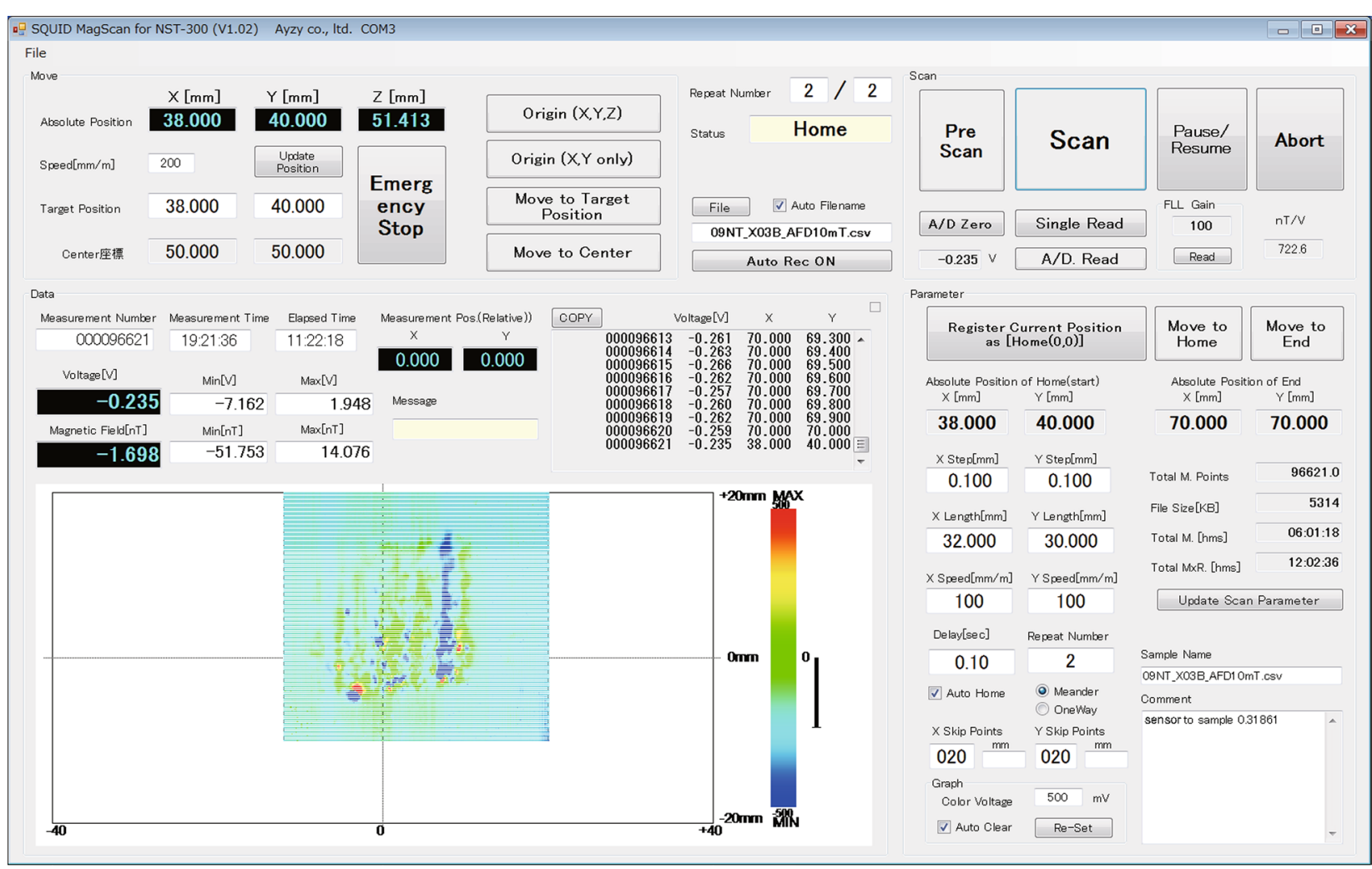

Fig. 5 Screenshot of measurement software SQUID MagScan 


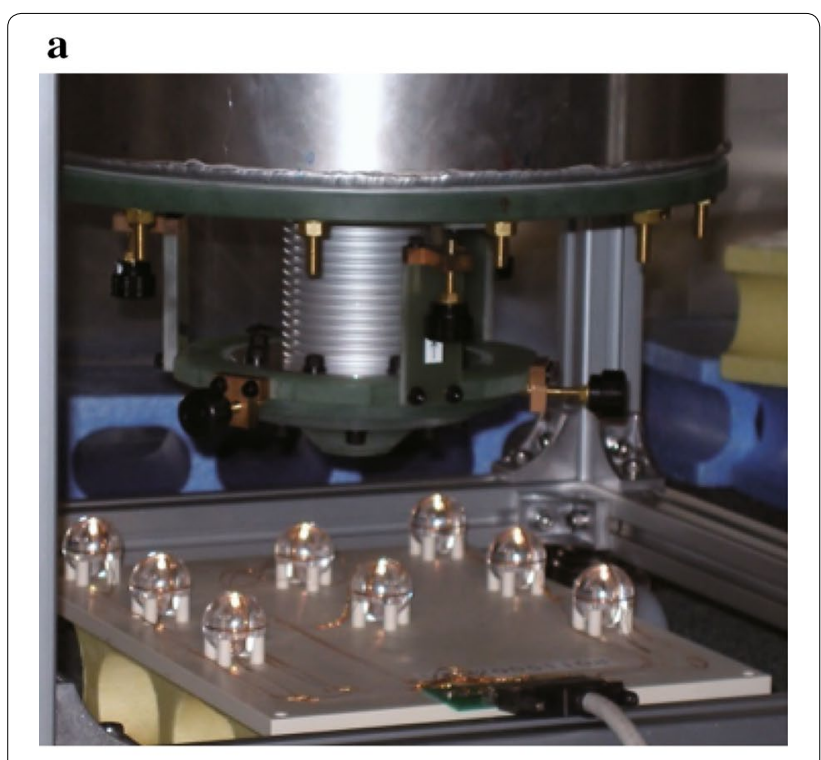

\section{b}

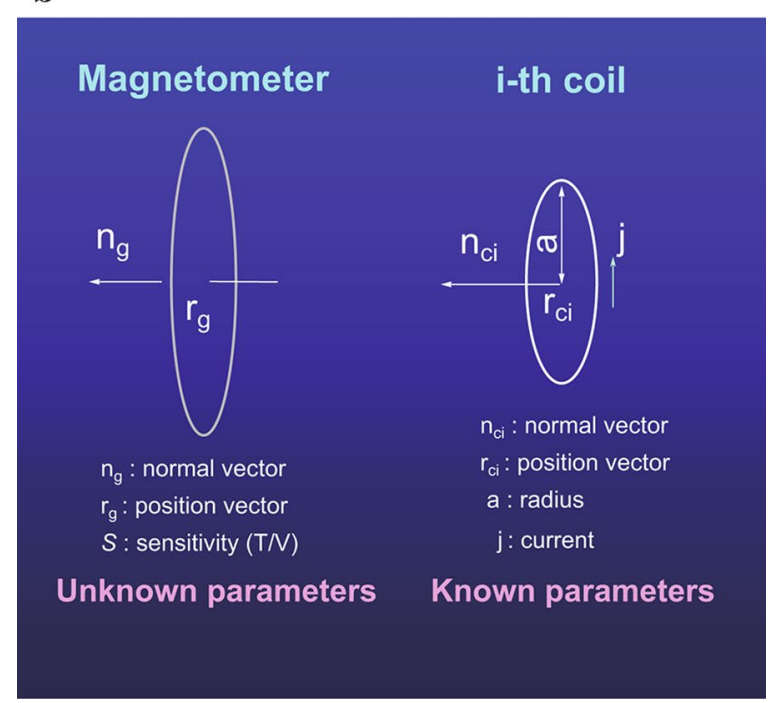

Fig. 6 Calibration of a SQUID sensor for the SQUID microscope. a Circular Current Array System. b Schematic diagram of calibration coils and the sensor

Adachi et al. (2014). It consists of eight 30-mm-diameter spheres on a plate and each sphere contains three coils that are perpendicular to each other. The $X-, Y$-, and $Z$-axes of the outer six spheres are parallel to each other. The three spheres in each of the outer rows are separated by $10 \mathrm{~cm}$, and the two outer rows are separated by $20 \mathrm{~cm}$. On the other hand, the coils on the $X$ - and $Y$-axes of the inner two spheres are set at a $45^{\circ}$ angle relative to the axes of the other six spheres. The SQUID sensor was placed $\sim 10 \mathrm{~cm}$ from the inner spheres.

A precise 10-mA AC current was supplied to each coil at $80 \mathrm{~Hz}$. The current control unit switched the current from one coil to another in sequence, and the SQUID sensor voltage was measured and stored for further analysis. Figure $6 \mathrm{~b}$ presents the known parameters (normal vectors $n_{c_{i}}$, position vectors $r_{c_{i}}$, radius $a$, and current $j$ of the magnetic field-generating coils) and the unknown parameters (normal vector $n_{g}$, position vector $r_{g}$, and sensitivity $S$ of the SQUID magnetometer pickup coil) used to estimate the sensitivity of the SQUID sensor (see Higuchi et al. 1989). The best estimate of the sensitivity was $722.6 \mathrm{nT} / \mathrm{V}$ (Kawai et al. 2016). The same sensitivity is applied to all SQUID sensors with the same design fabricated at KIT.

\section{Sensor-to-sample distance measured with a line current}

A line current was used to estimate the sensor-to-sample distance using a method similar to that of Fong et al. (2005). Figure $7 \mathrm{a}$ shows the straight $25-\mu \mathrm{m}$ aluminum wire glued onto the glass plate that is attached to the sample holder to be scanned. The current flows from the right (blue cable) to the left (white cable) typically with a DC current of $1.000 \mathrm{~mA}$.

Figure $7 \mathrm{~b}$ shows the interface of SQUID LineScan software, which was developed using MATLAB (MathWorks, Natick, MA, USA) for estimating the distance between the SQUID sensor and the line (or sample) using data collected from the current scan. The parameters whose values are required by the software to estimate the distance are listed on the right-hand side of the software user interface and include the thickness of the sapphire window, thickness of the film on the wire and on the sample, diameter of the wire, and amplitude of the current used for the scans. The polarity of SQUID, which can be flipped depending on the wiring of the SQUID chip on the sapphire rod, is also required. One set of line current scans usually includes three parallel scans $10 \mathrm{~mm}$ apart. Each line current scan is optimized to search for the right combination of SQUID-to-sample distance $d$ (approximately equal to half the distance between the maximum and minimum), the SQUID offset $s$, the conversion factor $c$ (close to 1 if the calibration is accurate), and the position shift $p$ along the scan direction that minimizes the difference between the line scan data and the data modeled according to Eq. 1:

$$
B_{i}=s+c \frac{\mu_{0} I\left(y_{0}+p+y_{i}\right)}{2 \pi\left[d^{2}+\left(y_{0}+p+y_{i}\right)^{2}\right]}
$$

where $B_{i}$ is the magnetic field [in teslas (T)], $c$ is the conversion factor, $s$ is the SQUID offset, $p$ is position shift along the $y$ grid position, $d$ is the distance between the line and the SQUID sensor, $y_{i}$ is the $y$ grid position, $y_{0}$ is the $y$ grid position where $B_{i}=0, I$ is the current amplitude; and $\mu_{0}$ is a constant $\left(4 \pi \times 10^{-7} \mathrm{~T} \mathrm{~m} / \mathrm{A}\right.$. The SQUID 
a

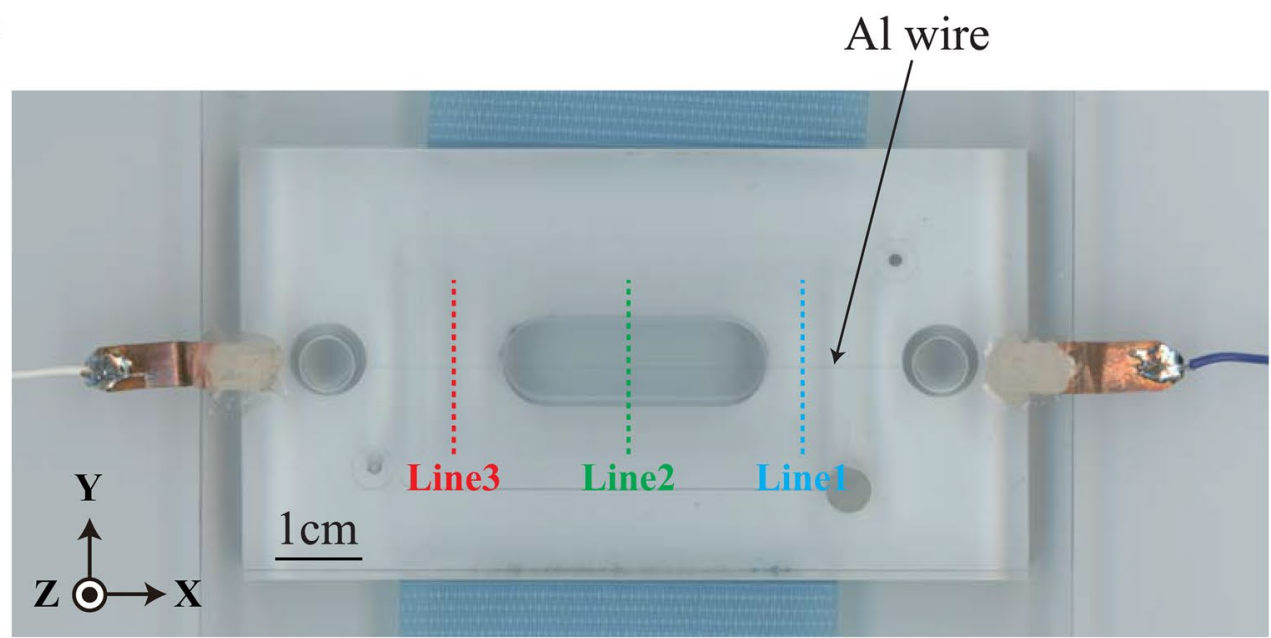

b

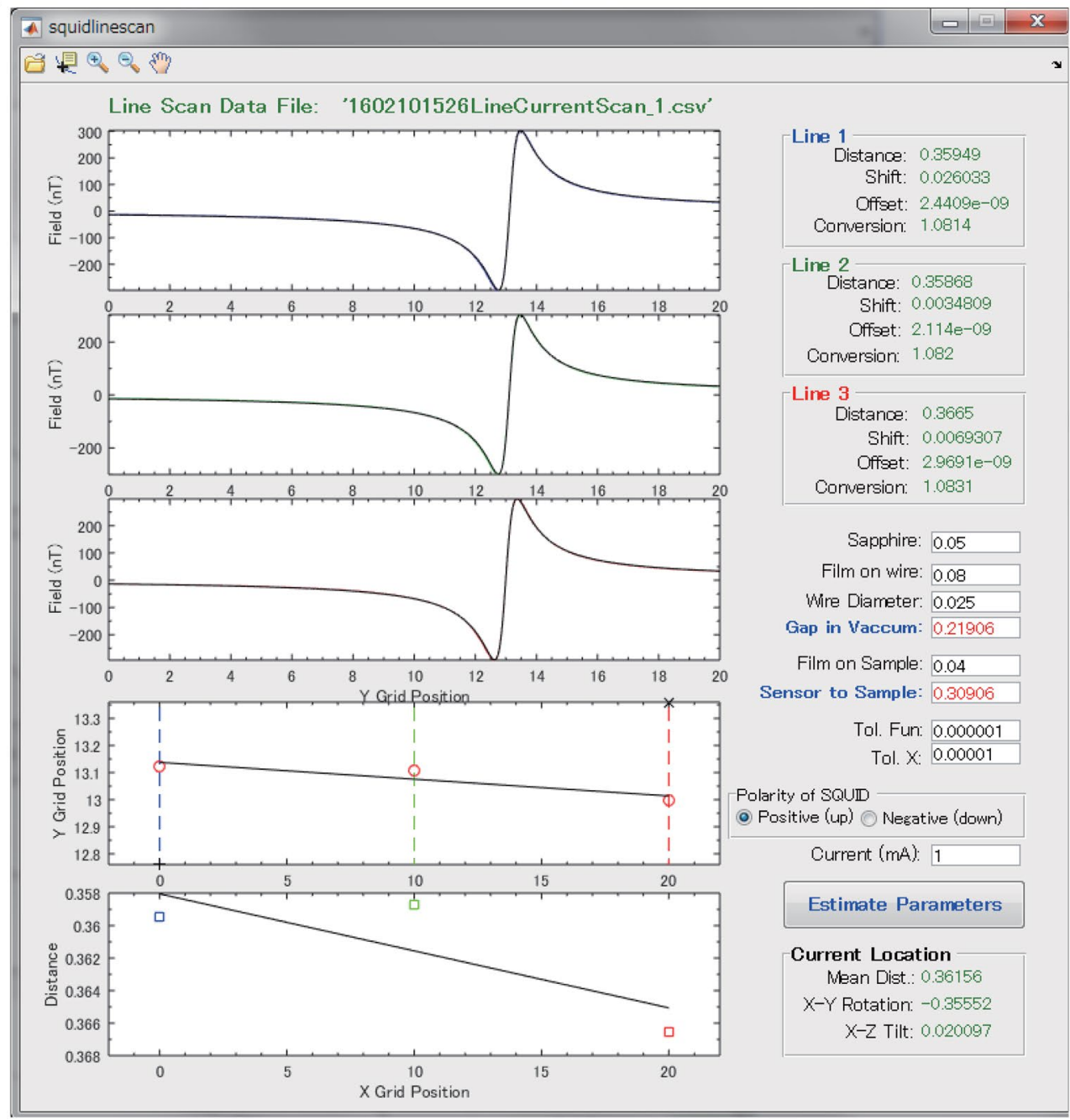

Fig. 7 a Line current measurement equipment. Aluminum wire (25 $\mu \mathrm{m}$ thick) is tightly stretched on a glass plate and covered with $80-\mu m$-thick plastic film (not shown here). b Screenshot of the SQUID LineScan program for the calculation of sensor-to-sample distance 
LineScan software reports the optimized values for each line scan, an average sensor-to-sample distance, and the gap length in a vacuum (i.e., distance between the sensor and the inner wall of the sapphire window).

Figure 8 shows the conversion factor as a function of sensor-to-sample distance estimated by a series of line current scans with increasing distance. The conversion factor approaches 1.00 as the distance increases to $\sim 10 \mathrm{~mm}$. The discrepancy between the actual value and the theoretical value at smaller distances (i.e., the conversion factors are further away from 1.0) might be related to the variability of the magnetic field produced by a line current within the $200-\mu \mathrm{m} \times 200-\mu \mathrm{m}$ coil. In fact, the magnetic flux distribution of a vertical magnetic point source measured with a square SQUID loop depends on the position along the diagonal [e.g., see Figs. 20 and 21 of Granata and Vettoliere (2016)].

\section{Noise and detection limit}

The noise characteristics of the SQUID microscope system were measured at $\sim 10-\mathrm{Hz}$ intervals without moving the $X Y Z$ stage in the MSB. Figure 9a shows the background noise of the SQUID magnetometer measured on July 7, 2015, from 12:00 p.m. to 1 p.m. A long-term drift component of more than $1 \mathrm{nT}$ is seen together with quasiperiodic fluctuations of about $60-90 \mathrm{~s}$. Figure $9 \mathrm{~b}$ shows the background noise measured on July 9, 2015, from 12:00 p.m. to 1 p.m. Although the quasiperiodic fluctuations of about 60-90 s are still observed, the drift component is less than that in Fig. 9a. The drift component might originate mainly from the change in the magnetic field of the Earth; however, long-term environmental

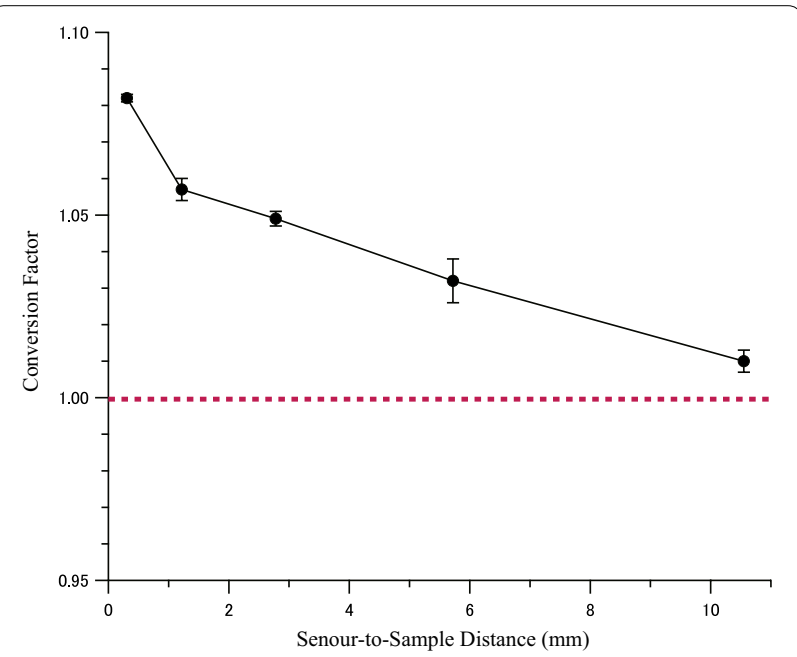

Fig. 8 Conversion factor as a function of sensor-to-sample distance. Error bar represents standard deviation of three values of sensor-tosample distances calculated for lines 1 through 3 magnetic fields resulting from activities in the laboratory could also be a source of the drift. The 60- to 90-s quasiperiodic component also could originate from within or near the laboratory. In addition, it is possible that the fluctuations in temperature or magnetic field are caused by air-conditioning or other components in or near the laboratory. This possibility will be investigated and improved in the future.

Figure 9c, d is expanded views of a 30-s period in Fig. 9a, b, respectively. The standard deviation of these short-period fluctuations is about 40-50 pT. Figure 9e shows the background noise of the SQUID sensor itself measured in a superconducting magnetic shield for $30 \mathrm{~s}$ at a sampling rate of $100 \mathrm{~Hz}$ with a low-pass filter (LPF) frequency of $10 \mathrm{~Hz}$ at KIT (Kawai et al. 2016). The standard deviation of $3.0 \mathrm{pT}$ is more than one order of magnitude smaller than that of the background noise of the SQUID microscope system. The low noise level for the SQUID sensor is achieved by the presence of the superconducting magnetic shield, which could be the minimum ideal system noise in the future.

The curves in Fig. 10 indicate the detection limit of a vertical dipole pointing up or down with a magnetic moment of $1 \times 10^{-14} \mathrm{Am}^{2}$ (blue line), $1 \times 10^{-15} \mathrm{Am}^{2}$ (yellow line), or $1 \times 10^{-16} \mathrm{Am}^{2}$ (purple line), assuming that $100 \mathrm{pT}$ is the minimum noise level (see Fig. 9c, d). For the typical sensor-to-sample distance (i.e., $\sim 200 \mu \mathrm{m}$ ) in current SQUID system, a magnetic moment of $1 \times 10^{-14} \mathrm{Am}^{2}$ can be detected if the long-term drift and 60- to 90-s quasiperiodic fluctuations are reduced and compensated. Considering the typical scanning interval $([X, Y]=[100 \mu \mathrm{m} \times 100 \mu \mathrm{m}])$ and the scanning rate of $\sim 0.36 \mathrm{~s} /$ point (e.g., number of points $[X, Y]=[651,301]$, delay time before data acquisition $[0.1 \mathrm{~s}]$, speed of motor $[X, Y]=[100 \mathrm{~mm} / \mathrm{min} ., 100 \mathrm{~mm} / \mathrm{min}$.], time from start to end $[70,440 \mathrm{~s}])$, one scan line in $Y$ direction takes $\sim 108 \mathrm{~s}$. This is shorter than the typical wave lengths of long-term drift observed in Fig. 9c, d, which can be reduced by the drift correction assuming linear drift and zero magnetic field at start and end of each line. For a stoichiometric magnetite (saturation magnetization $=\sim 4.8 \times 10^{5} \mathrm{~A} / \mathrm{m}$ ), a magnetic moment of $1 \times 10^{-14} \mathrm{Am}^{2}$ corresponds to an $\sim 275$-nm cube of magnetite. Reducing the sensor-tosample distance to $100 \mu \mathrm{m}$ allows detection of a magnetic moment of $1 \times 10^{-15} \mathrm{Am}^{2}$, which corresponds to an $\sim 128$-nm cubic magnetite. This sensitivity is comparable to the best possible moment sensitivity of $>10^{-15}$ $\mathrm{Am}^{2}$ estimated by Weiss et al. (2007a) for their SQUID microscope with a sensor-to-sample distance of 100 $\mu \mathrm{m}$. Further, if we achieve $1 \times 10^{-16} \mathrm{Am}^{2}$ corresponding to an $~ 59-\mathrm{nm}$ cube of magnetite, it is enough for most paleomagnetic studies because the SP/SD boundary of magnetite is around 15-50 nm (e.g., Newell and Merrill 

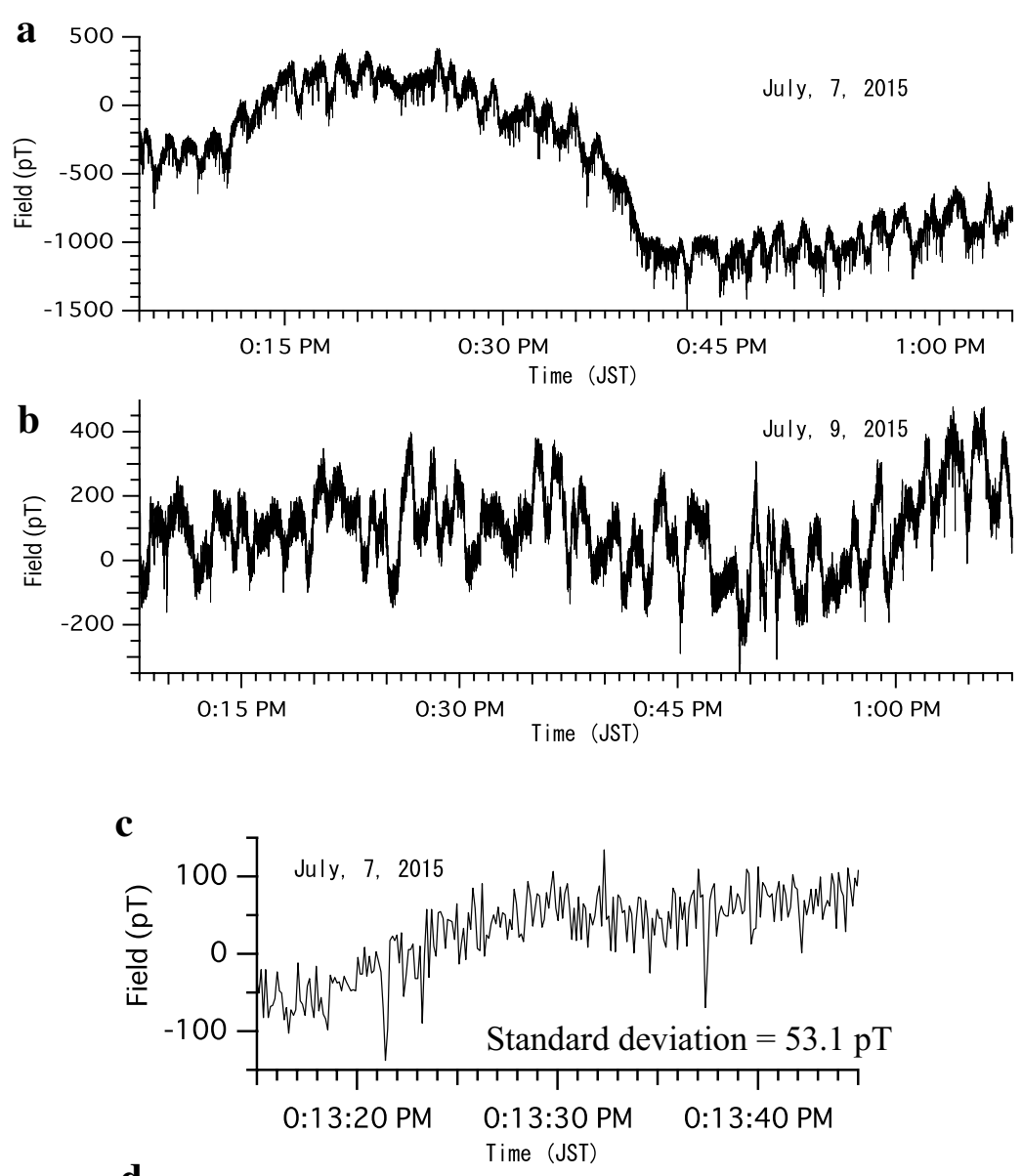

d
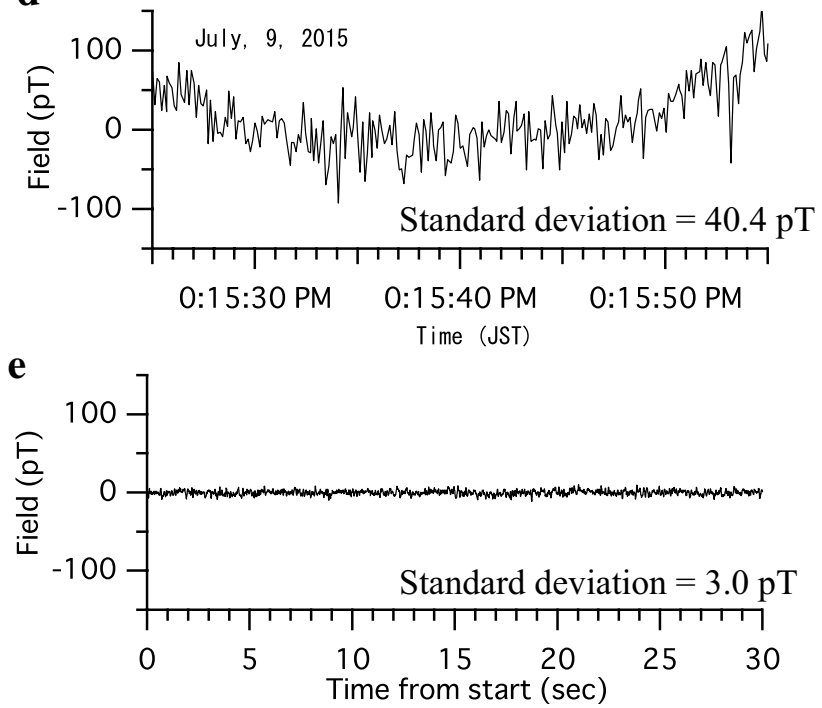

Fig. 9 Background noise of SQUID magnetometer measured on a July 7, 2015, and b July 9, 2015, for 1 h. In both recordings, a long-term drift component ( $>1 \mathrm{nT}$ in $\mathbf{a}$ ) is seen together with the periodic fluctuations of about 60-90 s. c, d Expanded 30-s measurement from $\mathbf{a}$ and $\mathbf{b}$, respectively. e Background noise of the SQUID sensor itself measured in a superconducting magnetic shield for $30 \mathrm{~s}$ at a sampling rate of $100 \mathrm{~Hz}$ with an LPF of $10 \mathrm{~Hz}$ 


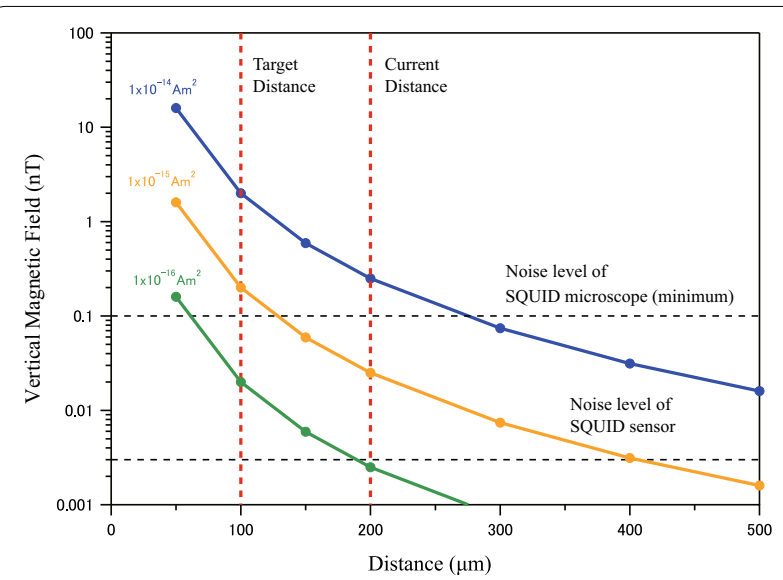

Fig. 10 Detection limit expected for a magnetic dipole. Magnetic fields produced just above a vertical magnetic dipole with magnetic moments of $1 \times 10^{-14} \mathrm{Am}^{2}$ (blue), $1 \times 10^{-15} \mathrm{Am}^{2}$ (yellow), and $1 \times 10^{-16} \mathrm{Am}^{2}$ (purple) are plotted versus distance. Red dashed vertical lines are the current sensor-to-sample distance $(200 \mu \mathrm{m})$ and the target distance $(100 \mu \mathrm{m})$, respectively. Minimum estimates of the noise level of the SQUID microscope system and the SQUID sensor are indicated by black horizontal dashed lines

1999). On the other hand, we may need to think about the stability of magnetic moment carried by a sample with small magnetic moment (Berndt et al. 2016). Kirschvink et al. (2015) reported the best sensitivity for a $2 \mathrm{G}$ SRM is $\sim 10^{-13} \mathrm{Am}^{2}$ using ultraclean quartz glass sample holder. The measurement of a small specimen, such as a magnetic inclusion in a single crystal (e.g., Sato et al. 2015; Tarduno et al. 2015), can be improved by using a SQUID microscope for measuring the magnetic moment. Recently, Lima and Weiss (2016) demonstrated that their SQUID microscope could measure a magnetic dipole moment down to about $10^{-15} \mathrm{Am}^{2}$.

\section{Precision of the $X Y$ stage positioning}

To evaluate the repeatability and accuracy of the sample positioning of the $X Y Z$ stage, we performed laser interferometry (Fig. 11a) with the laser encoder unit RLU10 (Renishaw plc, Gloucestershire, UK), similar to the measurements conducted by Oda et al. (2016b). The laser beam reflector (RLR10-A3-XF) was attached to the sample table, and the laser detector head (RLD 90 RRI) was tightly affixed to a tripod on the floor. While the sample table of the $X Y Z$ stage was moving, the $Y$ positions were monitored and recorded (the photograph in Fig. 11a is taken in the $\mathrm{X}$ direction for demonstration). The measurements were adjusted for the environmental conditions with temperature of $24^{\circ} \mathrm{C}$, humidity of $46 \%$, and atmospheric pressure of $99.86 \mathrm{kPa}$.

The plots in Fig. 11b show the difference between the position setting of the $X Y Z$ stage and the position
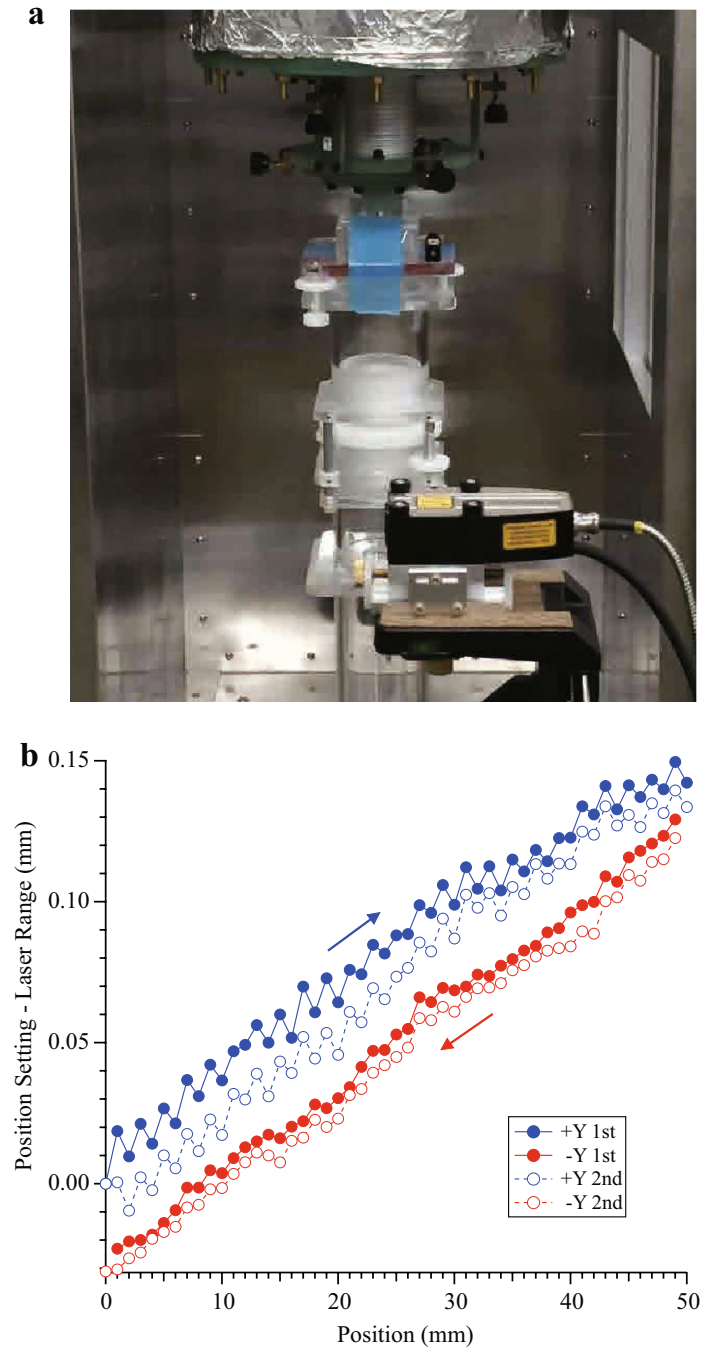

Fig. 11 a Experimental setup for laser interferometry measurement of the $X Y Z$ stage positioning. $\mathbf{b}$ Results of laser interferometry. $X$-axis stepping motor was fixed, and the $Y$-axis stepping motor was moved in 1-mm steps according to the software. Blue solid (open) circles plot the difference between the $X Y Z$ stage position setting and the position measured with laser interferometry in $+Y$ direction for the first (second) measurement, and the red solid (open) circles are for the $-Y$ direction measurements

determined by laser interferometry for the first (solid symbols) and second (open symbols) measurements. With the $X$-axis position fixed, the $X Y Z$ stage was moved in $+Y$ direction (blue symbols) from the starting position (zero) to $50 \mathrm{~mm}$ in 1-mm steps and then in $-Y$ direction (red symbols) back to the original position in 1-mm steps. There was a systematic increase (in $+Y$ direction) or decrease (in $-Y$ direction) of about $0.15 \mathrm{~mm}$ in the difference between the set position of the $X Y Z$ stage and that measured by laser interferometry at $50 \mathrm{~mm}$. This increase or decrease corresponds to a $0.3 \%$ systematic 
error or a $0.3^{\circ}$ deflection of the axis of the light relative to the $Y$-axis of the $X Y Z$ stage. In all cases, the random error of the $Y$ position was less than $\pm 0.01 \mathrm{~mm}$. The systematic difference of $\sim 0.03 \mathrm{~mm}$ between the $+Y$ measurements (blue) and the $-Y$ measurements (red) might have been caused by backlash from a ball screw contacting a ball nut. In conclusion, spacial resolution of the system is $\sim 200 \mu \mathrm{m}$, considering the size of pick-up coil, sensor-tosample distance, and the accuracy of $X Y Z$ stage discussed above.

\section{Two-axis tumbling AF demagnetization system for thin section samples}

Figure 12a shows the specially designed tumbler that rotates a thin section sample along two axes simultaneously during AF demagnetization. A thin section is covered with cotton for protection and is placed diagonally in the plastic inner rotator (the semitransparent piece on the left). The rotation axis of the inner rotator is supported by the two blue stands on the outer rotator. The spin of the motor beneath the blue pulley (left-hand side of Fig. 12b) is transmitted to the outer rotator via a urethane belt (dark orange component in Fig. 12b). While

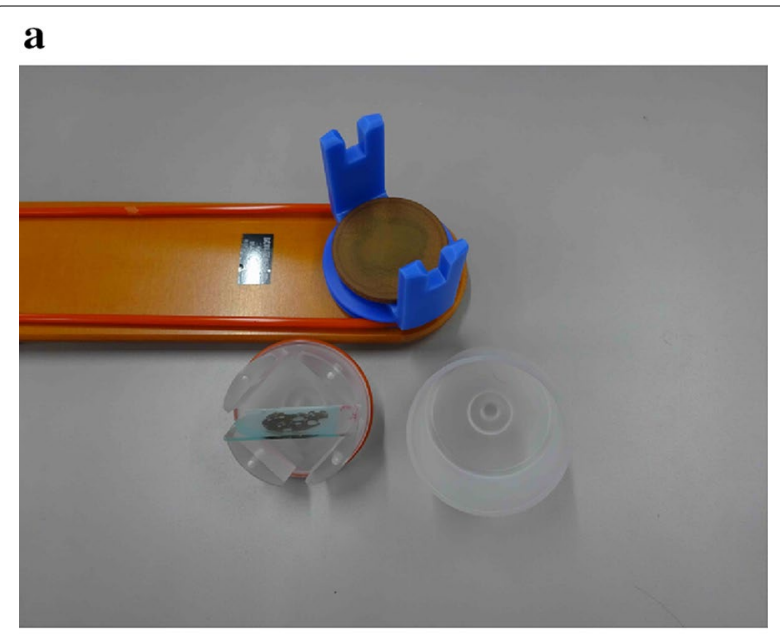

\section{b}

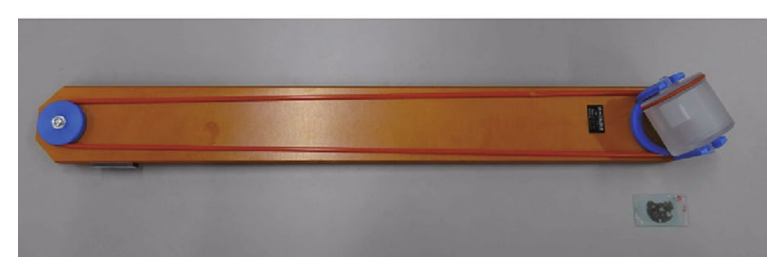

Fig. 12 a Sample holder and tumbler that enables rotation of a thin section sample along two axes simultaneously during AF demagnetization. b Sample holder and tumbler prepared for AF demagnetization the outer rotator spins along vertical axis, the inner rotator spins along the horizontal axis via the fixed circular pad (light brown piece in the outer rotator in Fig. 12a) that touches the thin urethane belt around the outside of the inner rotator. The tumbler rotates the thin section along two axes simultaneously at different rates, resulting in the random positioning of the thin section during AF demagnetization. AF demagnetization up to $100 \mathrm{mT}$ was performed using AF demagnetizer model DEM-95C (Natsuhara Co. Ltd.) with a three-layered PC permalloy shield in a residual magnetic field of $\sim 10 \mathrm{nT}$.

\section{Magnetic image registration using paired point sources \\ Principles}

It is usually not difficult to correlate positions on optical and electron microscopic images of thin sections. On the other hand, it is difficult to correlate the positions on those images with a magnetic image because the latter is blurred due to the distance from the magnetic source and is shifted horizontally depending on the direction of magnetization. Fong et al. (2005) demonstrated the registration of a magnetic image with a photographic image by using two line currents that perpendicularly crossed each other. Two crossed wires were placed on the sample holder after removing the thin section, and the magnetic image was taken. The magnetic image of a thin section was matched to that produced by the two crossed lines, which correlated with the optical images of the lines and with the optical image of the thin section. This method is effective for registering images; however, the magnetic measurement of the two crossed line currents requires additional setup and is time-consuming. Thus, we have developed an alternative method that uses two point sources placed diagonally on the sample holder outside of the thin section. The advantage of this method is that the sample and the point sources can be scanned at the same time.

\section{Demonstration of image registration}

A thin section of an Hawaiian basalt was pressed upside down onto the glass window of a flatbed optical color scanner (EPSON GT-X980 with a resolution of 6400 $\mathrm{dpi} \times 6400 \mathrm{dpi}$ and pixel size $=4 \mu \mathrm{m} \times 4 \mu \mathrm{m}$ ), covered with a thin transparent film for protection, and scanned using reflected light (Fig. 13a). The sample holder has two holes to anchor the point sources. We fabricated a sputtered FeCo circle, $75 \mu \mathrm{m}$ in diameter and $500 \mathrm{~nm}$ thick, on a $\mathrm{Si}$ substrate (see an optical microscopic image in Fig. 13e). Because each point source is fabricated in the middle of the Si chip, the center of the Si chip (Fig. 13a; point where the yellow lines cross) represents the position of the point source. Because the point sources are 


\section{a}
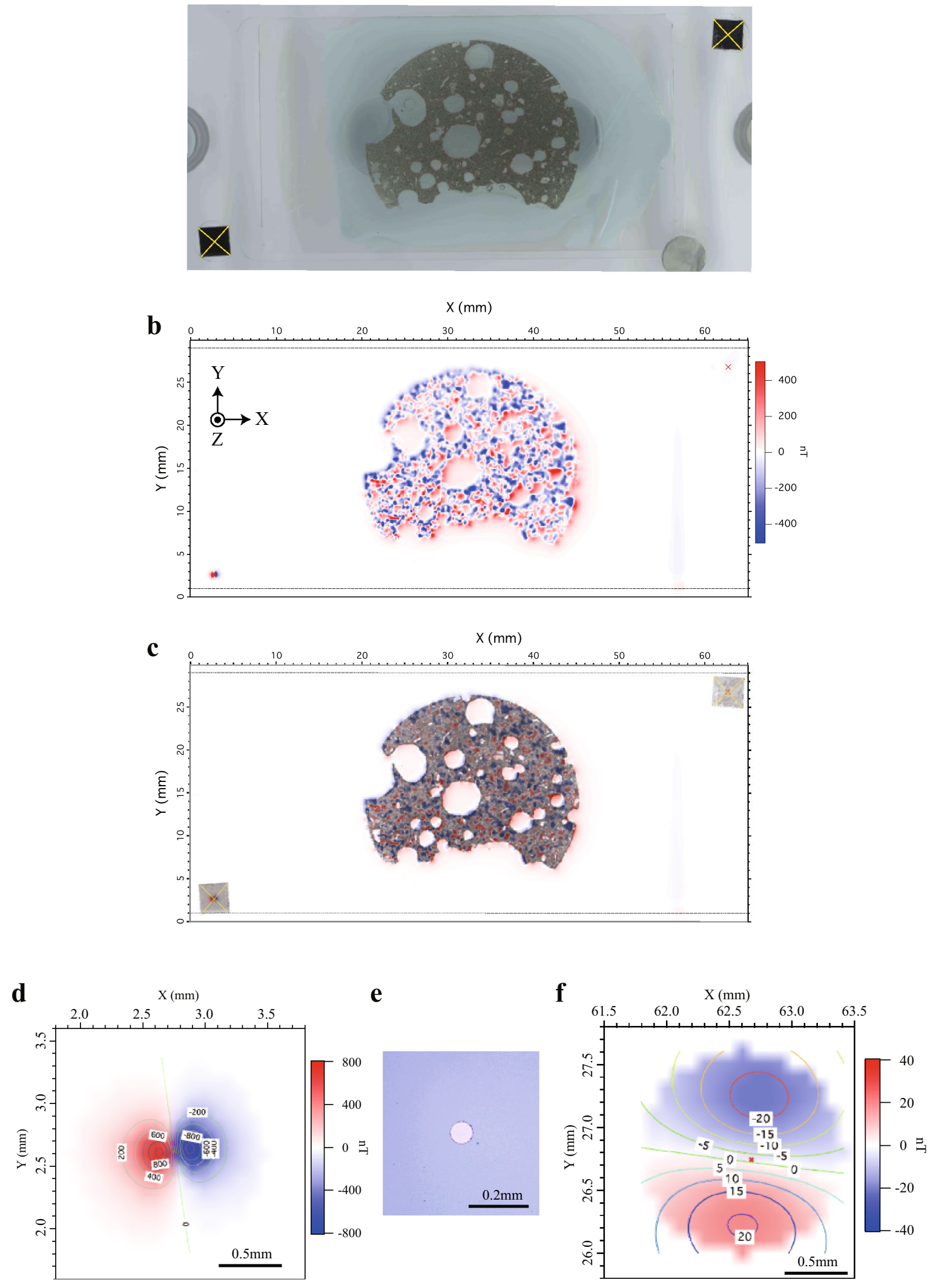
(See figure on previous page.)

Fig. 13 Registration of a magnetic image with an optical image using two point sources. Sensor-to-sample distance was $216 \mu \mathrm{m}$ when the magnetic field was measured. a Optical scanner image of a basalt thin section sample with two point sources on the corners. b Magnetic image of the same sample with NRM after 20-mT AFD. c Magnetic image overlaid on the gray-scaled optical image. $\mathbf{d}$ The image is an enlargement of the magnetic field produced by point source A placed in the lower-left corner of $\mathbf{b}$ outside of a thin section. The contours represent the magnetic field estimated for an optimized magnetic dipole after a nonlinear least-squares fit. The red cross in the center indicates the estimated position of the dipole on the XY plane. e Optical microscopic image of a $75-\mu \mathrm{m} \varphi$ point source made of FeCo on a Si square substrate. $\mathbf{f}$ The image is an enlargement of the magnetic field produced by point source B in the upper-right corner of $\mathbf{b}$. Explanations on contours and the red cross are the same as $\mathbf{d}$

flat disks, they can be easily magnetized in the horizontal direction.

Figure 13b shows the magnetic image of natural remanent magnetization (NRM) of the basalt sample measured after AF demagnetization at $20 \mathrm{mT}$ peak field. The color bar on the right gives the magnetic field values in nT; e.g., red and blue correspond to positive and negative values, respectively. We applied linear drift correction for the raw data assuming averages of the first and the last ten measurements (above and below the two horizontal broken lines in Fig. 13b) for each line scan in the $+Y$ direction (starting at $0 \mathrm{~mm}$ and ending at $50 \mathrm{~mm}$ ) should be adjusted to zero. Figure 13c overlays the magnetic image in Fig. 13b on the optical image in Fig. 13a after conversion to a gray-scale image. Leakage of the magnetic field in the bubble holes within the sample and outside of the sample can be observed.

To register the magnetic image relative to the optical image, the positions of the point sources are calculated by fitting the dipolar magnetic field to the theoretical distribution of a dipole. Figure $13 \mathrm{~d}$ is an enlargement $(2 \mathrm{~mm} \times 2 \mathrm{~mm})$ of the magnetic image produced by point source A on the lower-left corner in Fig. 13b. To eliminate the noise from sources other than the point sources (e.g., dust particles), the magnetic data from only within $0.8 \mathrm{~mm}$ (the distance limit parameter can be changed) of the expected center (i.e., the midpoint of the maximum and the minimum for a horizontal dipole) were used to fit the data. We used the "Curve fitting" feature of the program IGOR Pro (WaveMetrics, Inc., Lake Oswego, OR, USA) to find the best dipole fit. A user-defined function in IGOR Pro produced a vertical magnetic field for a magnetic dipole calculated using the following formula:

$$
\boldsymbol{B}(r)=\frac{\mu_{0}}{4 \pi\|r\|^{3}}\left(\frac{3 r(\boldsymbol{m} \cdot \boldsymbol{r})}{\|\boldsymbol{r}\|^{2}}-\boldsymbol{m}\right)
$$

where $\boldsymbol{r}=(x, y, z)-\left(x_{0}, y_{0}, z_{0}\right)$ and $\boldsymbol{m}=\left(m_{x}, m_{y}, m_{z}\right)$, where $(x, y, z)$ is the measurement position, $\left(x_{0}, y_{0}, z_{0}\right)$ is the dipole position, and $\left(m_{x}, m_{y}, m_{z}\right)$ is the magnetic moment of a dipole. A nonlinear least-squares data fit using the Levenberg-Marquardt algorithm was used to search for the minimum value of residual sum of squares. An initial guess for $\left(x_{0}, y_{0}\right)$ was the midpoint between the maximum and minimum for an assumed horizontal dipole, which is located manually using a cursor on the horizontal map. An initial guess for the $z_{0}$ position (depth) was set at $-0.3 \mathrm{~mm}$ (approximately the sensorto-sample distance), and $m_{x}, m_{y}$, and $m_{z}$ were set to zero.

The search for the minimum value of residual sum of squares began by traveling downhill from the starting point on the surface of residual sum of squares. We conducted several "Curve fitting"s with the default of 40 iterations, where each "Curve fitting" started with the previously estimated optimum values until no improvement was observed.

The contours in Fig. 13f represent the magnetic field produced by an optimized magnetic dipole after a nonlinear least-squares fit. The red cross $(\mathbf{x})$ in the center indicates the estimated position of the dipole on the $X Y$ plane. Figure $13 \mathrm{f}$ is an enlargement of the magnetic field produced by point source $B$ in the upper-right corner of Fig. 13b. Because the Si chip of point source B was placed upside down, the signal is smaller and broader than that of the point source A.

Table 1 gives the results of the magnetic dipole fitting of the two point sources for six consecutive measurements of a basalt thin section. All $X$ and $Y$ positions were measured relative to a fixed home position. The standard deviation of the $Y$ position was $\sim 0.5 \mathrm{~mm}$ for the $X$-axis and $\sim 0.35 \mathrm{~mm}$ for the $Y$-axis. The vertical distance of point sources A and B from the sensor was $0.29 \pm 0.02 \mathrm{~mm}$ and $0.91 \pm 0.16 \mathrm{~mm}$, respectively. The difference in the vertical distance was due to point accidentally placing source $\mathrm{B}$ underneath the Si chip, which was $\sim 0.5 \mathrm{~mm}$ thick. The standard deviation of the difference in the $X$ and $Y$ positions of the two point sources was 0.09 and $0.10 \mathrm{~mm}$, respectively. The results indicate that the relatively large standard deviation of the estimated horizontal position might be caused not by calculation error but by the relative shift between the sample holder and the thin section, because a rubber cylinder presses the sample in a slightly different manner on each sample change (see circular component in the lower-right corner of Fig. 13a).

Figure $14 \mathrm{a}-\mathrm{d}$ demonstrates the change in the magnetic image depending on the direction of magnetization of a thin section. Figure 14a is the magnetic image after subjecting a thin section to anhysteretic remanent 


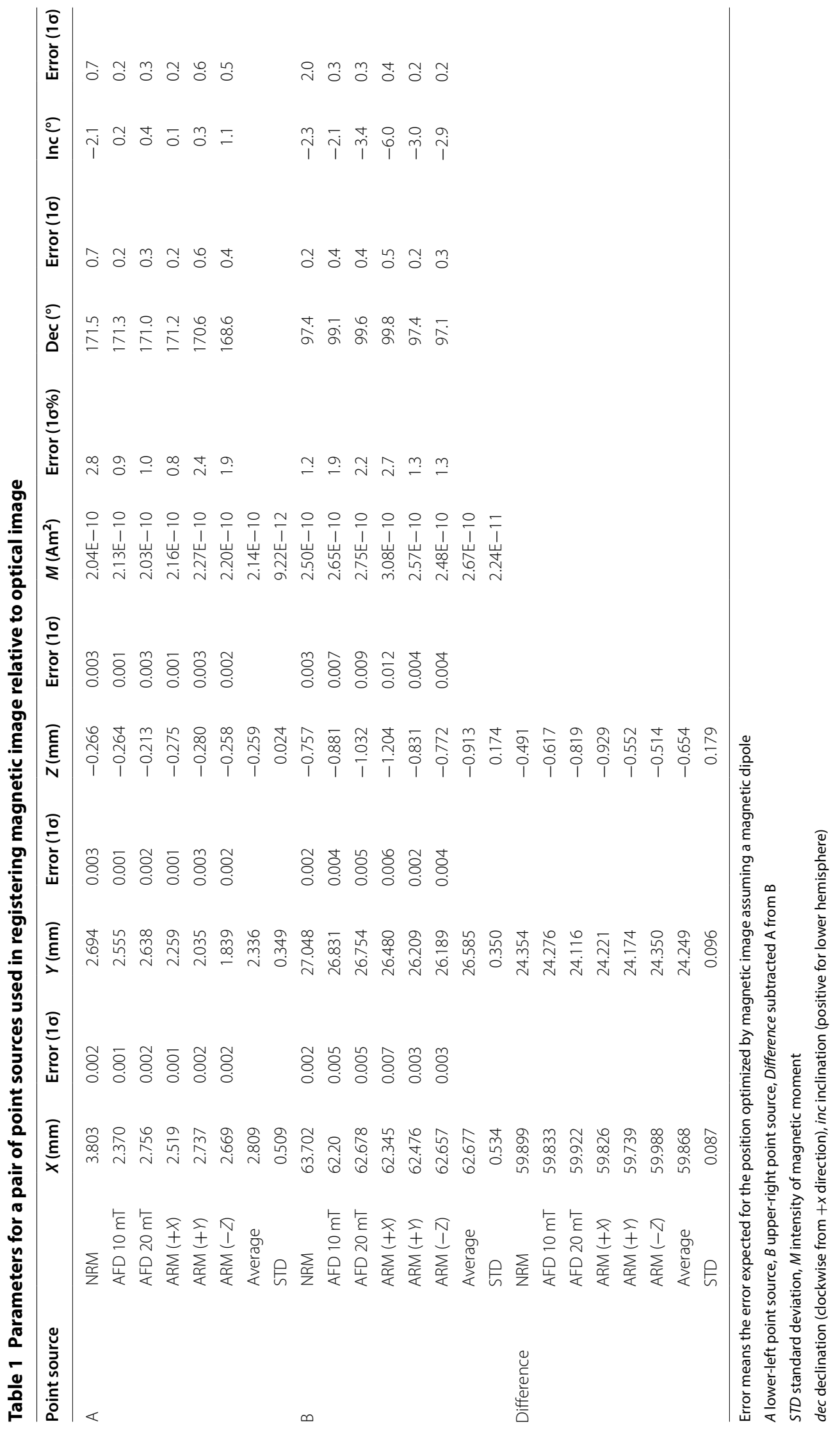




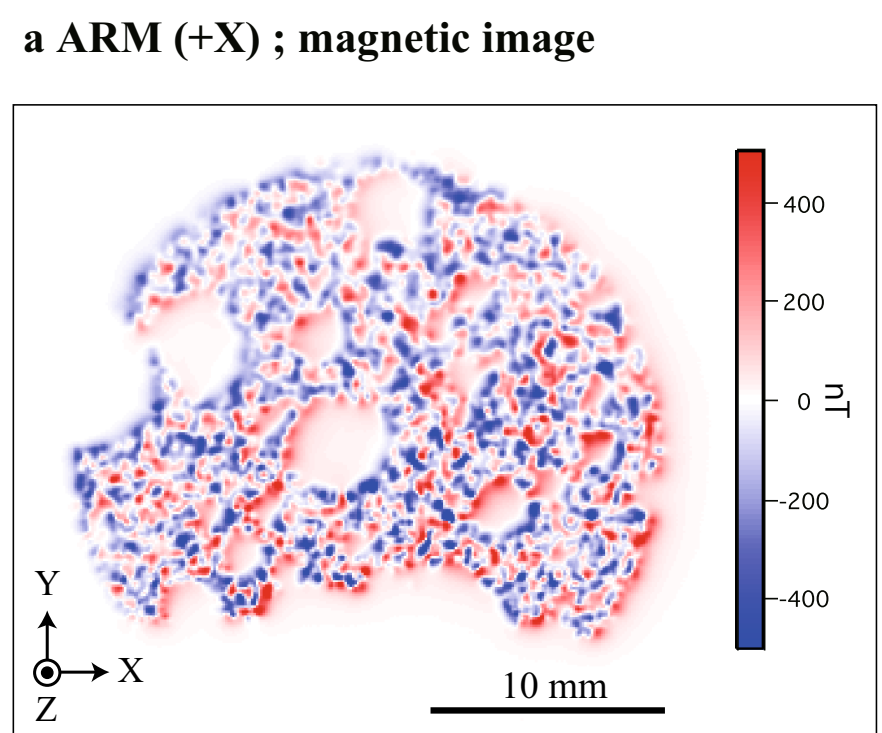

\section{c ARM (-Z) ; magnetic image}

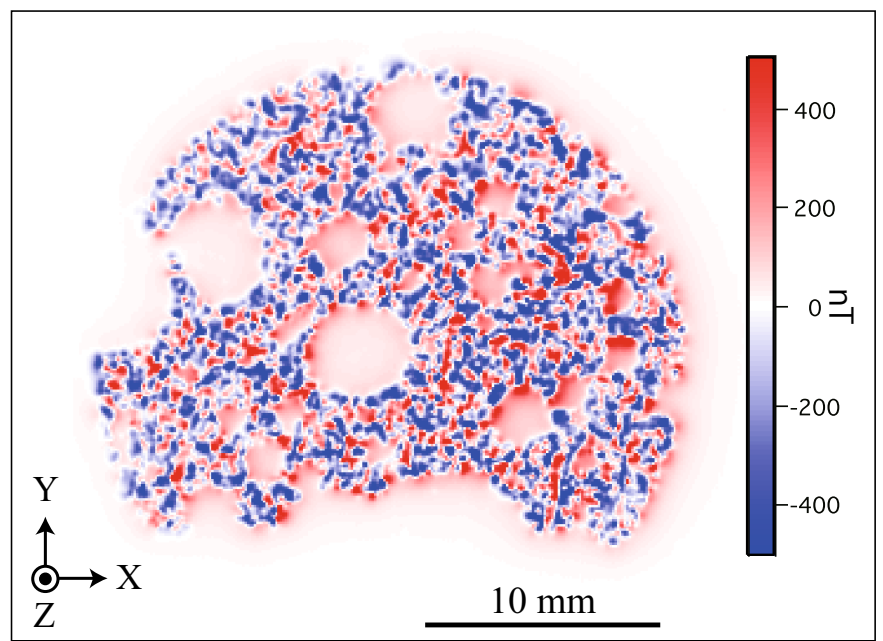

\section{b ARM (+X) ; overlay image}

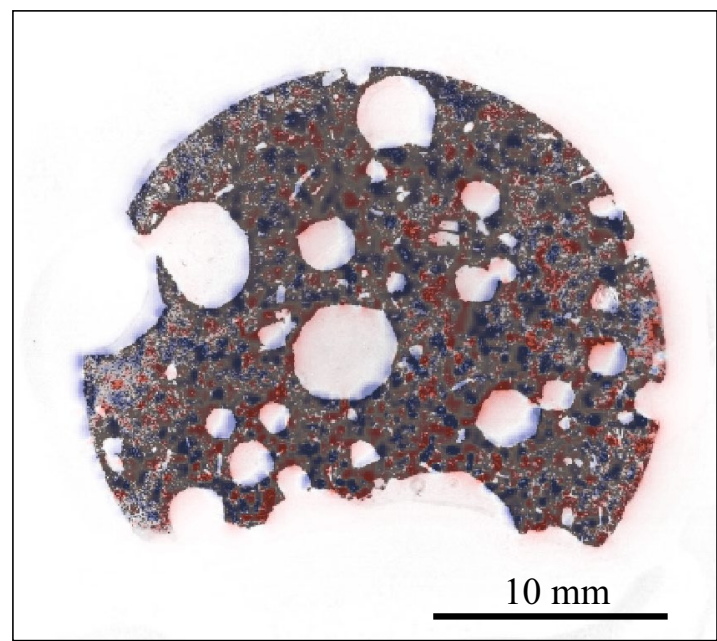

d ARM (-Z) ; overlay image

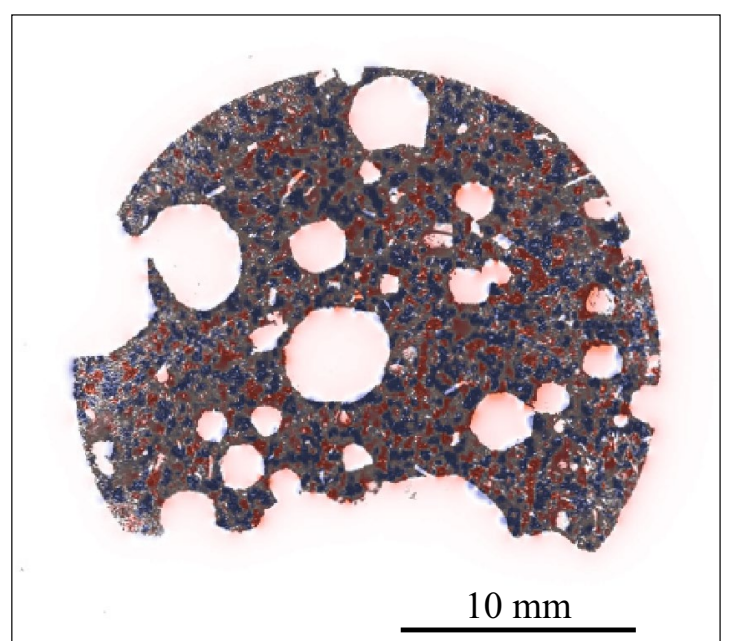

Fig. 14 Magnetic images after subjecting the thin section sample to ARM with a DC field of $50 \mu T$ and an AC field of $80 \mathrm{mT}$. a Magnetic image of ARM in the $+X$ direction. $\mathbf{b}$ Image in a overlaid on the optical scanner image. c Magnetic image of ARM in the $-Z$ direction. $\mathbf{d}$ Image in $\mathbf{c}$ overlaid on the optical scanner image. Color scale represents the vertical magnetic field in nT (positive upward)

magnetization (ARM) $(\mathrm{DC} 50 \mu \mathrm{T}, \mathrm{AC} 80 \mathrm{mT})$ in the $+X$ direction. Figure 14b is the result of overlaying Fig. 14a on the gray-scaled optical scanner image. The positive magnetic field is observed on the right-hand side of the sample optical image and the negative magnetic field on the left-hand side. This is consistent with the direction of sample magnetization. Figure $14 \mathrm{c}$ is the magnetic image after subjecting a thin section to the same ARM as in Fig. 14a but in the $-Z$ direction. Figure $14 \mathrm{~d}$ is the result of overlaying Fig. 14c on the gray-scaled optical scanner image.

Magnetic field leaks outside of the sample area or in the bubble holes appear as positive values in general, which is consistent with the overall negative magnetization of a sample. These results demonstrate the successful application of our new method of registering a thin section sample using a pair of point sources placed on the sample holder. 


\section{Conclusions}

We developed a scanning SQUID microscope system for imaging the vertical component of a magnetic field on the surfaces of geological samples at room temperature. The system comprises a SQUID microscope, a magnetically shielded box (MSB), an $X Y Z$ stage, and auxiliary electronic equipment. The SQUID sensor is a washer-type pick-up coil with $200 \mu \mathrm{m} \times 200 \mu \mathrm{m}$, which has an inner hole of $30 \mu \mathrm{m} \times 30 \mu \mathrm{m}$. The SQUID microscope can operate up to 4 days on $10 \mathrm{~L}$ of liquid He. The MSB is made of two-layered PC permalloy with shielding factors of $1 / 257,1 / 288$, and $1 / 91$ in the $X, Y$, and $Z$ directions, respectively. The DC component of residual magnetic field at the measurement position was less than $5 \mathrm{nT}$. The non-magnetic $X Y Z$ stage with its $80-\mathrm{cm}$ neck is driven by three-axis stepping motors underneath the MSB. The stroke of the $X Y Z$ stage is $100 \mathrm{~mm}$ in the $X$ and $Y$ directions and has an accuracy of $\sim 10 \mu \mathrm{m}$ confirmed by laser interferometry. The easy-to-use graphical software SQUID MagScan controls the $X Y Z$ stage and digitizes the analog voltage output from the FLL connected to SQUID sensor while scanning a thin section.

A Circular Current Array System calibrated the sensitivity of the SQUID sensor as $722.6 \mathrm{nT} / \mathrm{V}$. The sensor-tosample distance was estimated by scanning a precision line current and analyzing the results using the developed SQUID LineScan software. We achieved the minimum sensor-to-sample distance of $\sim 200 \mu \mathrm{m}$ by using a $50-\mu \mathrm{m}$-thick sapphire window and a $40-\mu \mathrm{m}$ thin protective film on a thin section sample. Considering the size of pick-up coil, sensor-to-sample distance, and the accuracy of $X Y Z$ stage, spacial resolution of the system is $\sim 200 \mu \mathrm{m}$. The minimum noise level of a SQUID microscope is expected to be $\sim 100 \mathrm{pT}$, excluding long-term drift and fluctuations, which is more than an order of magnitude larger than that of a SQUID sensor $(\sim 3 \mathrm{pT})$. With the current minimum sensor-to-sample distance of $\sim 200 \mu \mathrm{m}$ and a minimum noise level of $\sim 100 \mathrm{pT}$, a magnetic dipole with a magnetic moment of $1 \times 10^{-14} \mathrm{Am}^{2}$ can be detected; this corresponds to an $\sim 275$-nm cube of magnetite.

We proposed a new method for registering a magnetic image with an optical image that uses a pair of point sources placed diagonally on the sample holder outside of the thin section sample. We performed six successful demonstrations of this method using a basalt thin section and found a standard deviation of $\sim 0.1 \mathrm{~mm}$ for a distance ( $x 59.9 \mathrm{~mm}, y 24.2 \mathrm{~mm}$ ) between the two point sources. Magnetic images of the NRM and ARM overlaid on the gray-scaled optical image are comprehensive and could be used for further analysis, modeling, and interpretation.

\section{Abbreviations}

AC: alternating current; ADC: analog-to-digital converter; AF: alternating field; AIST: National Institute of Advanced Industrial Science and Technology; ARM: anhysteretic remanent magnetization; DC: direct current; FLL: flux-locked loop; GFRP: glass fiber-reinforced plastics; GSJ: Geological Survey of Japan; KIT: Kanazawa Institute of Technology; LPF: Iow-pass filter; LSB: least significant bit; NRM: natural remanent magnetization; SQUID: superconducting quantum interference device; SRM: SQUID rock magnetometer.

\section{Authors' contributions}

$\mathrm{HO}$ designed and developed the entire system and wrote and edited most of the manuscript; JK developed the SQUID sensor and designed the sapphire window and rod and other components of the SQUID microscope; MM developed the electronics (FLL) for the SQUID sensor; IM designed the sample holder; MS conducted measurements and interpreted the results; AN conducted measurements and maintenance; YY conducted measurements on the range of the laser and provided the samples; JF developed the cryostat for the SQUID microscope; NN designed and developed the $X Y Z$ stage and tumbler; YA developed the $X Y Z$ stage controller and data acquisition software; TM designed the magnetic shield box; $C X$ developed the software for the analysis of the line scan data and edited the manuscript. All authors read and approved the final manuscript.

\section{Author details}

${ }^{1}$ Research Institute of Geology and Geoinformation, Geological Survey of Japan, AIST, Central 7, 1-1-1 Higashi, Tsukuba 305-8567, Japan. ${ }^{2}$ Applied Electronics Laboratory, Kanazawa Institute of Technology, Kanazawa 920-1331, Japan. ${ }^{3}$ Research Institute of Earthquake and Volcano Geology, Geological Survey of Japan, AIST, Central 7, 1-1-1 Higashi, Tsukuba 305-8567, Japan. ${ }^{4}$ Center for Advanced Marine Core Research, Kochi University, B200 Monobe, Nankoku, Kochi 783-8502, Japan. ${ }^{5}$ Fujihira Co. Ltd., Tsukuba 305-0047, Japan. ${ }^{6}$ Natsuhara Giken Co. Ltd., Osaka 532-0033, Japan. ${ }^{7}$ Ayzy Co. Ltd., Kyoto 616-8312, Japan. ${ }^{8}$ Ohtama Co., Ltd., 1744 Oshitate, Inagi, Tokyo 206-0811, Japan. ${ }^{9}$ School of Ocean and Earth Science, National Oceanography Centre Southampton, University of Southampton, Waterfront Campus, European Way, Southampton SO14 3ZH, UK.

\section{Acknowledgements}

Hirokuni Oda was supported by a JSPS Grant-in-Aid for Scientific Research (A) (Funding No. 25247073). Chuang Xuan is supported by a startup fund provided by the University of Southampton. JSPS provided a Visiting Fellowship for Foreign Researchers (Award No. PE14034) for Chuang Xuan to visit the Geological Survey of Japan (GSJ), a part of the National Institute of Advanced Industrial Science and Technology (AIST), to conduct work related to this research. The authors are indebted to Norihiro Nakamura, Yoichi Usui, and Akira Usui for contributing to the discussions on the system. The magnetically shielded box, sapphire rod with metalized wire, and sapphire window were made by Ohtama Co., Ltd., Kyocera Corporation, and Ceraken Co., Ltd., respectively. Tomomi Kobayashi helped in the development of convenient tools necessary for the SQUID microscope system. The authors thank Ayako Katayama for performing the measurements with the scanning SQUID microscope system and producing the artwork. The authors thank Miki Kawabata for fabricating and evaluating the SQUID chips. The manuscript was improved by the positive comments of the two anonymous reviewers and the editor.

\section{Competing interests}

The authors declare that they have no competing interests.

Received: 30 July 2016 Accepted: 15 October 2016

Published online: 12 November 2016

\section{References}

Adachi Y, Higuchi M, Oyama D, Haruta Y, Kawabata S, Uehara G (2014) Calibration for a multichannel magnetic sensor array of a magnetospinography system. IEEE Trans Magn 50:5001304

Baudenbacher F, Peters NT, Wikswo JP Jr (2002) High resolution low-temperature superconductivity superconducting quantum interference device microscope for imaging magnetic fields of samples at room temperature. Rev Sci Instrum 73:1247-1254 
Baudenbacher F, Fong LE, Holzer JR (2003) Monolithic low-transition-temperature superconducting magnetometers for high resolution imaging magnetic fields of room temperature samples. Appl Phys Lett 82:3487-3489

Berndt T, Muxworthy AR, Fabian K (2016) Does size matter? Statistical limits of paleomagnetic field reconstruction from small rock specimens. J Geophys Res Solid Earth 121:15-26. doi:10.1002/2015JB012441

Chatraphorn S, Fleet EF, Wellstood FC (2000) Scanning SQUID microscopy of integrated circuits. Appl Phys Lett 76:2304-2306

Constable C, Parker R (1991) Deconvolution of longcore palaeomagnetic measurements - spline therapy for the linear problem. Geophys J Int 104:453-468. doi:10.1111/j.1365-246X.1991.tb05693.x

Fischer WW, Fike DA, Johnson JE, Raub TD, Guan Y, Kirschvink JL, Eiler JM (2014) SQUID-SIMS is a useful approach to uncover primary signals in the Archean sulfur cycle. Proc Natl Acad Sci 111:5468-5473. doi:10.1073/ pnas.1322577111

Fong LE, Holtzer JR, McBride KK, Lima EA, Baudenbacher F (2005) High-resolution room-temperature sample scanning superconducting quantum interference device microscope configurable for geological and biomagnetic applications. Rev Sci Instrum 76:053703

Fu RR, Weiss BP, Lima EA, Harrison RJ, Bai XN, Desch SJ, Ebel DS, Suavet C, Wang H, Glenn D, Sage DL, Kasama T, Walsworth RL, Kuan AT (2014) Solar nebula magnetic fields recorded in the Semarkona meteorite. Science 346:1089-1092. doi:10.1126/science.1258022

Fu RR, Weiss BP, Lima EA, Kehayias P, Araujo JFDF, Glenn DR, Gelb J, Einsle JF, Bauer AM, Harrison RJ, Ali GAH, Walsworth RL (2016) Evaluating the paleomagnetic potential of single zircon crystals using the Bishop Tuff. Earth Planet Sci Lett. doi:10.1016/j.epsl.2016.09.038

Gattacceca J, Boustie M, Weiss BP, Rochette P, Lima EA, Fong LE, Baudenbacher FJ (2006) Investigating impact demagnetization through laser impacts and SQUID microscopy. Geology 34:333-336. doi:10.1130/G21898.1

Granata C, Vettoliere A (2016) Nano superconducting quantum interference device: a powerful tool for nanoscale investigations. Phys Rep 614:1-69. doi:10.1016/j.physrep.2015.12.001

Higuchi M, Chinone K, Ishikawa N, Kado H, Kasai N, Nakanishi M, Koyanagi M, Ishibashi Y (1989) The positioning of magnetometer pickup coil in dewar by artificial signal source. In: Wiliamson SJ (ed) Advances in biomagnetism. Plenum Press, New York, pp 701-704

Jackson M, Bowles JA, Lascu I, Solheid P (2010) Deconvolution of u channe magnetometer data: experimental study of accuracy, resolution, and stability of different inversion methods. Geochem Geophys Geosyst 11:Q07Y10. doi:10.1029/2009GC002991

Kawai J, Oda H, Fujihira J, Miyamoto M, Miyagi I, Sato M (2016) SQUID microscope with hollow-structured cryostat for magnetic field imaging of room temperature samples. IEEE Trans Appl Supercond 26:1600905. doi:10.1109/TASC.2016.2536751

Kirschvink JL, Isozaki Y, Shibuya H, Otofuji Y, Raub TD, Hilburn IA, Kasuya T, Yokoyama M, Bonifacie M (2015) Challenging the sensitivity limits of paleomagnetism: magnetostratigraphy of weakly magnetized Guadalupian-Lopingian (Permian) limestone from Kyushu, Japan. Palaeogeogr Palaeoclimatol Palaeoecol 418:75-89

Kirtley JR, Wikswo JP Jr (1999) Scanning SQUID microscopy. Annu Rev Mater Sci 29:117-148

Lima EA, Weiss BP (2016) Ultra-high sensitivity moment magnetometry of geological samples using magnetic microscopy. Geochem Geophys Geosyst. doi:10.1002/2016GC006487

Lima EA, Bruno AC, Carvalho HR, Weiss BP (2014) Scanning magnetic tunnel junction microscope for high-resolution imaging of remanent magnetization fields. Meas Sci Technol 25:105401

Newell AJ, Merrill RT (1999) Single-domain critical sizes for coercivity and remanence. J Geophys Res 104(B1):617-628. doi:10.1029/1998JB900039

Oda H, Xuan C (2014) Deconvolution of continuous paleomagnetic data from pass-through magnetometer: a new algorithm to restore geomagnetic and environmental information based on realistic optimization. Geochem Geophys Geosyst 15:3907-3924. doi:10.1002/2014GC005513

Oda H, Usui A, Miyagi I, Joshima M, Weiss BP, Shantz C, Fong LE, McBride KK, Harder R, Baudenbacher F (2011) Ultrafine-scale magnetostratigraphy of marine ferromanganese crust. Geology 39:227-230

Oda H, Miyagi I, Kawai J, Suganuma Y, Funaki M, Imae N, Mikouchi T, Matsuzaki T, Yamamoto Y (2016a) Volcanic ash in bare ice south of Sor Rondane Mountains, Antarctica: geochemistry, rock magnetism and nondestructive magnetic detection with SQUID gradiometer. Earth Planets Space 68:39. doi:10.1186/s40623-016-0415-3

Oda H, Xuan C, Yamamoto Y (2016b) Toward robust deconvolution of passthrough paleomagnetic measurements: new tool to estimate magnetometer sensor response and laser interferometry of sample positioning accuracy. Earth Planets Space 68:109. doi:10.1186/s40623-016-0493-2

Ono Y, Ishiyama A (2004) Development of biomagnetic measurement system for mice with high spatial resolution. Appl Phys Lett 85:332-334

Sato M, Yamamoto S, Yamamoto Y, Okada Y, Ohno M, Tsunakawa H, Maruyama S (2015) Rock-magnetic properties of single zircon crystals sampled from the Tanzawa tonalitic pluton, central Japan. Earth Planets Space 67:150. doi:10.1186/s40623-015-0317-9

Tarduno JA, Cottrell RD, Davis WJ, Nimmo F, Bono RK (2015) A Hadean to Paleoarchean geodynamo recorded by single zircon crystals. Science 349:521-524. doi:10.1126/science.aaa9114

Wang Q, Qin H, Liu Q, Song T (2014) Room temperature sample scanning SQUID microscope for imaging the magnetic fields of geological specimens. Appl Mech Mater 475-476:3-6

Weiss BP, Kirschvink JL, Baudenbacher FJ, Vali H, Peters NT, Macdonald FA, Wikswo JP Jr (2000) A low temperature transfer of ALH84001 from Mars to Earth. Science 290:791-795

Weiss BP, Lima EA, Fong L, Baudenbacher F (2007a) Paleomagnetic analysis using SQUID microscopy. J Geophys Res 112:B09105

Weiss BP, Lima EA, Fong LE, Baudenbacher F (2007b) Paleointensity of the Earth's magnetic field using SQUID microscopy. Earth Planet Sci Lett 264:61-71

Xuan C, Oda H (2015) UDECON: deconvolution optimization software for restoring high-resolution records from pass-through paleomagnetic measurements. Earth Planets Space 67:183. doi:10.1186/ s40623-015-0332-x

Yoshida T, Higuchi M, Komuro T, Kado H (1994) Calibration system for a multichannel SQUID magnetometer. In: Proceedings of the 16th annual international conference of IEEE engineering in medicine and biology society. Engineering advances: new opportunities for biomedical engineers, November 1994, pp 171-172

\section{Submit your manuscript to a SpringerOpen ${ }^{\odot}$ journal and benefit from:}

- Convenient online submission

- Rigorous peer review

- Immediate publication on acceptance

- Open access: articles freely available online

- High visibility within the field

- Retaining the copyright to your article

Submit your next manuscript at springeropen.com 